\title{
Topological signatures in CMB temperature anisotropy maps
}

\author{
W. S. Hipólito-Ricaldi and G. I. Gomero \\ Instituto de Física Teórica, Universidade Estadual Paulista, Rua Pamplona 145 São Paulo, SP 01405-900, Brazil
}

(Received 19 October 2005; published 28 November 2005)

\begin{abstract}
We propose an alternative formalism to simulate cosmic microwave background (CMB) temperature maps in $\Lambda \mathrm{CDM}$ universes with nontrivial spatial topologies. This formalism avoids the need to explicitly compute the eigenmodes of the Laplacian operator in the spatial sections. Instead, the covariance matrix of the coefficients of the spherical harmonic decomposition of the temperature anisotropies is expressed in terms of the elements of the covering group of the space. We obtain a decomposition of the correlation matrix that isolates the topological contribution to the CMB temperature anisotropies out of the simply connected contribution. A further decomposition of the topological signature of the correlation matrix for an arbitrary topology allows us to compute it in terms of correlation matrices corresponding to simpler topologies, for which closed quadrature formulas might be derived. We also use this decomposition to show that CMB temperature maps of (not too large) multiply connected universes must show "patterns of alignment," and propose a method to look for these patterns, thus opening the door to the development of new methods for detecting the topology of our Universe even when the injectivity radius of space is slightly larger than the radius of the last scattering surface. We illustrate all these features with the simplest examples, those of flat homogeneous manifolds, i.e., tori, with special attention given to the cylinder, i.e., $T^{1}$ topology.
\end{abstract}

DOI: $10.1103 /$ PhysRevD.72.103008

PACS numbers: 98.70.Vc, 02.30.Gp, 98.80.Es, 98.80.Jk

\section{INTRODUCTION}

It is becoming widely recognized that universes with a nontrivial spatial topology may be more natural models for our Universe than the traditional simply connected ones. This naturalness can be invoked from the mathematical point of view by arguing that there is an infinity of locally homogeneous and isotropic multiply connected 3-spaces, while there are only three simply connected ones; or with physical arguments coming from incursions into the nobody's territories of quantum gravity and quantum cosmology. On the other hand, from a more pragmatic point of view, we can argue that cosmological models with a nontrivial spatial topology offer a very rich field of research, and are particularly well suited to explain certain reported "anomalous" features in cosmic microwave background (CMB) temperature maps, such as the alignments of their low $\ell$ modes [1,2].

Conversely, the full sky CMB temperature maps produced by the space missions COBE and WMAP provide us with an amazingly rich and high quality amount of data with which we can look for the topology of space. This is very compelling for those who wish to unmask our Universe and see its shape, since cosmic topology is at present an almost exclusively observational and phenomenological issue, due to the lack of an accepted fundamental physical theory which can predict the global topology of space.

Theory demands topology of space to leave several different kinds of marks in CMB temperature maps. Two

\footnotetext{
*Electronic address: hipolito@ift.unesp.br

†Electronic address: german@ift.unesp.br
}

of them have been largely studied and exploited to try to unveil the shape of our Universe, the distortion of the angular power spectrum with respect to that of a simply connected universe [3-7], and the existence of "circles in the sky" $[8,9]$. Two other closely related signatures, a nonnull bipolar power spectrum [10,11] and alignments of the low $\ell$ modes [1,2,7], have been only marginally used. Our main motivation for deciding to adventure into cosmic topology with the CMB was the desire to get a deeper understanding of the nature and properties of these alignments as a topological signature.

One indispensable tool for a project like this is a software facility to produce simulated CMB temperature maps in multiply connected universes, so that we could systematically study the effects of different sizes and topologies on these alignments. These simulation procedures exist and have been used in several studies in cosmic topology $[5,12-17]$, so we could expect that this issue of the project would not present any problem.

However, almost all known methods for computing CMB temperature anisotropies in multiply connected universes need to solve the Helmholtz equation in the manifold modeling our three-dimensional space, the only exception to our knowledge being the work of Bond et al. [17]. To solve the Helmholtz equation is a relatively easy problem in Euclidean manifolds [4,16], but a very difficult task in spherical $[18,19]$ and hyperbolic 3-spaces [20]. Indeed, the spherical case has been completely solved analytically only recently by Lachièze-Rey [19], while for compact hyperbolic manifolds the only possible approach is numerical [20].

Among other results, in this paper we present a new approach to the computation of the correlation matrix 
$\left\langle a_{\ell m} a_{\ell^{\prime} m^{\prime}}^{*}\right\rangle$ of the coefficients of the spherical harmonic decomposition of CMB temperature anisotropies in a universe with nontrivial spatial topology. The main feature of this approach is that it avoids the explicit computation of the solutions of the Helmholtz equation in the spatial sections of spacetime. Instead, we express the correlation matrix in terms of the covering group alone. Incidentally, the idea of generating a CMB map exploiting the symmetries of the quotient space was already suggested by Janna Levin and collaborators $[12,13]$. In particular, we wish to quote a citation of a nontrivial claim in [13] (page 2695) which we have, in our opinion, succeeded in achieving: "By understanding the symmetries of the fundamental polyhedron and the identification rules, a CBR pattern can be deduced without the need to explicitly obtain the spectrum mode by mode."

Our main formal result is a generic decomposition of the form

$$
X^{\Gamma}=X^{\text {s.c. }}+X^{\text {t.s. }} \text {, }
$$

where $X$ may be any covariance function which can be related to the two-point correlation function of the Newtonian potential (see Sec. III), $\Gamma$ is the covering group of (the multiply connected) space, s.c. stands for "simply connected," and t.s. means "topological signature."

Thus, in Eq. (1), $X^{\Gamma}$ is the covariance function computed in the manifold $M=\tilde{M} / \Gamma$, and $X^{\text {s.c. }}$ is the same covariance function but computed in the universal covering space $\tilde{M}$. It means that all the topological information is encoded in the "perturbative" term $X^{\text {t.s. }}$, and that is why we refer to it, generically, as the topological signature of $X^{\Gamma}$. This decomposition is always possible whenever one can express $X^{\Gamma}$ in terms of $\Gamma$, as, for example, in the pair separations histogram method for detecting multiple copies of standard candles [21]. In the present case, we succeeded in writing the correlation matrix of the $a_{\ell m}$ 's in this way by formally manipulating the two-point correlation function of the Newtonian potential derived by Bond, Pogosyan, and Souradeep [17].

Our approach to compute the correlation matrix $\left\langle a_{\ell m} a_{\ell^{\prime} m^{\prime}}^{*}\right\rangle$ has some methodological advantages in the simulation of CMB temperature maps. Indeed, by means of a suitable decomposition of the covering group $\Gamma$ in cyclic subgroups, we are able to write down a formula for the correlation matrix of a complicated topology in terms of the correlation matrices of the cyclic topologies (topologies with a cyclic covering group) that cover it maximally. Since correlation matrices for cyclic manifolds are relatively easy to compute (we obtain a closed quadrature formula for the cylinder), we expect to obtain in the near future more efficient ways to simulate maps for complicated manifolds.

The decomposition of $\Gamma$ in cyclic subgroups describes in a transparent way the symmetries of the manifold, and this fact gives rise to another advantage of our approach, in this case from the observational side. Universes with a cyclic topology present an alignment along the "direction of the generator isometry." It follows that a CMB map in a universe with a nontrivial topology might present "patterns of alignment" (one for each $\ell$ mode) characteristic of its shape and size. Indeed, and this shall become "obvious," symmetries of the quotient manifolds translate into symmetries of their patterns of alignment. We propose a method to search for these patterns by constructing maps of the dispersion of the squares $\left|a_{\ell m}\right|^{2}$ around the power spectrum. This opens the door to the development of methods to look for topology by searching these patterns, instead of limiting ourselves to considerations concerning only the special directions defined by the alignments.

For the sake of brevity, other advantages of our approach to compute the matrix $\left\langle a_{\ell m} a_{\ell^{\prime} m^{\prime}}^{*}\right\rangle$ are discussed in Sec. VI only. We prefer now to make a few remarks on some limitations of our work. We have considered here a few simplifications to develop the formalism, and worked out the details for the very simplest nontrivial topologies. In fact, we (i) have considered the Sachs-Wolfe effect as the only source of temperature anisotropies in CMB maps, (ii) have written the correlation matrix $\left\langle a_{\ell m} a_{\ell^{\prime} m^{\prime}}^{*}\right\rangle$ formally only for Euclidean 3-spaces, (iii) have worked out the details for homogeneous flat manifolds, (iv) have made a detailed analysis and some simulations only for cylinders ( $T^{1}$ topology), and (v) these simulations were done using the Einstein-de Sitter model. We wish to close this introduction by justifying each one of these simplifications.

The shape of space is a global property, thus we expect the topological signatures in CMB to show themselves on very large scales only. Although a proof is missing, we believe that the main features of these signals would observationally appear if we restrict the searches to the low $\ell$ modes in the temperature maps, i.e., we do not need high resolution CMB maps in cosmic topology. Since the SachsWolfe effect is the main source of temperature anisotropies at these scales [14], we expect that theoretical explorations considering only this effect will put in evidence the main features of the topological signals that we would observe in a real map. The addition of the missing part of the anisotropies will only modify quantitatively the predictions made with our approximation and, thus, will only be important when adjusting theoretical models with data.

We consider this paper technically hard, so much care has been taken to write it in a clear and pedagogical way. The main features of our formalism and of the topological signatures we predict in $\mathrm{CMB}$ can be understood by restricting the presentation to Euclidean topologies. The inclusion of nonzero spatial curvature will only introduce additional technical considerations (and nothing qualitatively new), thus we decided to leave the spherical and hyperbolic cosmological models for a future paper.

The same applies to the lack in the paper of detailed explicit correlation matrices for nonhomogeneous flat 
manifolds. Indeed, the price we pay for simplicity and transparency in the presentation of the results for each specific cyclic topology is the need for very hard calculations in the middle steps, as can be seen in the appendices. Explicit calculations for nonhomogeneous flat manifolds will only add one page to the main body of the paper, and one or two more appendices to the already large list of them (see the end of the introduction). An exhaustive presentation for all the Euclidean manifolds is left for a future work.

Last but not least, we performed the simulations with the Einstein-de Sitter model for simplicity. Nothing is lost from the theoretical point of view with this simplification since, as discussed in the paper, the structure of the topological signatures in CMB is captured in this oversimplified and old fashioned model of our Universe. However, more realistic $\Lambda \mathrm{CDM}$ models will be required to confront theory with observations quantitatively.

We close this introduction by giving a detailed description of the structure of the paper. In Sec. II we briefly review the two most common methods to simulate CMB temperature maps in multiply connected universes, as well as present the method we have developed, and implemented for the Euclidean case. In Sec. III we define the topological signature in a correlation function, perform the decomposition of the covering group of a quotient space in its cyclic subgroups, and write the topological signature in terms of this decomposition. We also show here that the symmetries of a quotient space appear transparently in the decomposition of its covering group in cyclic subgroups.

In Sec. IV we apply our formalism to the homogeneous Euclidean manifolds, which are the simplest. We first compute the correlation matrix and the angular power spectrum for the cylinder, and apply the general results of the previous section in order to write down the correlation matrix of the spherical harmonic coefficients and the angular power spectrum for a generic torus. Finally, as an illustration, we write those expressions explicitly for the chimney ( $T^{2}$ topology).

In Sec. V we first show, by means of simulations, that in a universe with the topology of a cylinder the low $\ell$ modes are aligned in a similar fashion as they are in the WMAP data. We then use the results in the previous sections to argue that $\mathrm{CMB}$ temperature maps in a universe with a nontrivial topology must present characteristic patterns of alignment, and propose the method of mapping on the sphere the dispersion of the squares $\left|a_{\ell m}\right|^{2}$ to look for them. Finally, in Sec. VI we discuss in detail the results of this paper and suggest further lines of research.

In brief, the main goal of this paper is to show that our approach to the computation of the correlation matrix $\left\langle a_{\ell m} a_{\ell^{\prime} m^{\prime}}^{*}\right\rangle$ (Sec. II), together with the decomposition of the covering group of a manifold in cyclic subgroups (Sec. III), led to the discovery of a new topological signature in $\mathrm{CMB}$ temperature maps, i.e., the "patterns of alignment" (Sec. V). To illustrate this we have used the simplest example, i.e., that of flat homogeneous manifolds (Sec. IV).

The paper has four appendices. In Appendix A we collect standard definitions and results related to spherical harmonics in order to set the conventions used in this paper. The technical calculations needed for the computation of the correlation matrix $\left\langle a_{\ell m} a_{\ell^{\prime} m^{\prime}}^{*}\right\rangle$ for the cylinder are presented in Appendices B and C. In the former, we develop from scratch the theory of Clausen $\varphi$ functions, for which we have not found any suitable reference in the literature. In the latter, we compute a function that is the key part for computing efficiently the topological signature of the correlation matrix for the cylinder. Finally, in Appendix D we briefly reproduce known results for the correlation matrix of five out of the six compact orientable Euclidean 3spaces. These formulas have been obtained previously in the literature by considering explicitly the solutions of the Helmholtz equation in these manifolds, and are written in terms of the $k$ modes $[4,16]$. Our derivation avoids the need for considering these solutions.

\section{SIMULATING CMB TEMPERATURE MAPS}

In this section we briefly describe two methods currently available for simulating CMB temperature maps in universes with nontrivial spatial topology, and proceed to develop our own formulation. We consider $\Lambda \mathrm{CDM}$ universes, where the background metric of spacetime is of the Robertson-Walker type, and include scalar and adiabatic perturbations as the seeds for the temperature anisotropies of the CMB. In the Newtonian gauge we have

$$
d s^{2}=a^{2}(\eta)\left[(1+2 \Phi) d \eta^{2}-(1-2 \Phi) \gamma_{i j} d x^{i} d x^{j}\right]
$$

for the metric, where $\eta$ is the conformal time, $a(\eta)$ is the scale factor, $\Phi$ is the Newtonian potential, and

$$
\gamma_{i j}=\left(1+\frac{K}{4}\left(x^{2}+y^{2}+z^{2}\right)\right)^{-2} \delta_{i j}
$$

is the metric of the spatial section of the background with sectional curvature $K=0, \pm 1$.

The matter content consists of radiation $\left(\Omega_{r}\right)$, baryonic and cold dark matter $\left(\Omega_{m}=\Omega_{b}+\Omega_{c d m}\right)$, and dark energy in the form of a cosmological constant $\left(\Omega_{\Lambda}\right)$. Since we are interested on fluctuations on large angular scales, we make the assumption of instantaneous recombination and do not consider finite thickness effects. The main contribution to the temperature anisotropy observed at the direction $\mathbf{n}$ comes from the complete (ordinary plus integrated) Sachs-Wolfe effect

$$
\frac{\delta T}{T}(\mathbf{n})=\frac{1}{3} \Phi\left(\eta_{\mathrm{LSS}}, R_{\mathrm{LSS}} \mathbf{n}\right)+\left.2 \int_{\eta_{\mathrm{LSS}}}^{\eta_{0}} d \eta \frac{\partial \Phi}{\partial \eta}\right|_{(\eta, R(\eta) \mathbf{n})},
$$

where the index LSS stands for "last scattering surface," 
the index 0 for present time, and $R(\eta)$ is the comoving distance at instant $\eta$ between a photon, scattered at $\eta_{\text {LSS }}$, and the observer.

The Newtonian potential is written as

$$
\Phi(\eta, \mathbf{x})=\int d q F(\eta, q) \xi(q, \mathbf{x}) .
$$

The temporal part satisfies the equation

$$
\begin{aligned}
& F^{\prime \prime}(\eta)+3 \mathcal{H}\left(1+c_{s}^{2}\right) F^{\prime}(\eta) \\
& \quad+\left[2 \mathcal{H}^{\prime}+\left(1+3 c_{s}^{2}\right)\left(\mathcal{H}^{2}-K\right)+c_{s}^{2} q^{2}\right] F(\eta)=0,
\end{aligned}
$$

where $c_{s}$ is the speed of sound in the fluid and $\mathcal{H}=a^{\prime} / a$ is the Hubble parameter in conformal time. On the other hand, the spatial part consists of solutions of the Helmholtz equation

$$
\left(\Delta+q^{2}\right) \xi(q, \mathbf{x})=0,
$$

where the index $q$ has been put as a variable in $\xi$ for simplicity of notation.

The integral in Eq. (3) has to be understood in a measure theoretic sense. Indeed, for multiply connected spaces the measure $d q$ is not the usual one but a combination of a discrete and an absolutely continuous measure, reducing the integral in (3) to a sum and an integral in the usual sense. In particular, if the space is compact, the measure reduces to a discrete one. This comes from the well-known fact that not every eigenmode of the Laplacian operator in the universal covering space $\tilde{M}$ is also an eigenmode in a quotient space $M=\tilde{M} / \Gamma$. In fact, only eigenmodes in $\tilde{M}$ satisfying the invariance conditions

$$
\xi(q, g \mathbf{x})=\xi(q, \mathbf{x})
$$

for any $g \in \Gamma$ project to eigenmodes in $M$.

The most straightforward way of simulating CMB temperature maps is by solving (4) and (5), performing the sum in (3), and then evaluating the SW effect (2). However, one has to consider that a temperature anisotropy map is a realization of a random field on the 2 -sphere, and this randomness is inherited from that of the Newtonian potential (3). There are currently two ways to implement this random character in the simulations, and one goal of this paper is to propose a third one.

The first and most direct method is to consider the randomness in the temporal part of the decomposition (3) of the Newtonian potential. The two-point correlation function of the Newtonian potential at fixed time $\eta$ can then be written as

$$
\left\langle\Phi(\eta, \mathbf{x}) \Phi\left(\eta, \mathbf{x}^{\prime}\right)\right\rangle=\int d q d q^{\prime} f\left(\eta, q, q^{\prime}\right) \xi(q, \mathbf{x}) \xi^{*}\left(q^{\prime}, \mathbf{x}^{\prime}\right),
$$

where $f\left(\eta, q, q^{\prime}\right)=\left\langle F(\eta, q) F\left(\eta, q^{\prime}\right)\right\rangle$ is the two-point correlation function for the amplitudes of the scalar perturbation modes and $\xi(q, \mathbf{x})$ are normalized solutions of the Helmholtz equation.
Assuming that the Newtonian potential is a homogeneous and isotropic random field, the two-point correlation function (7) reduces to a function of time $\eta$ and the distance $d\left(\mathbf{x}, \mathbf{x}^{\prime}\right)$, and thus we get $f\left(\eta, q, q^{\prime}\right)=$ $P_{\Phi}(\eta, q) \delta\left(q-q^{\prime}\right)$, where $P_{\Phi}(\eta, q)$ is the gravitational power spectrum. If, in addition, the Newtonian potential is assumed to be Gaussian, its random character is completely encoded in the variance of the temporal part

$$
\left\langle F^{2}(\eta, q)\right\rangle=P_{\Phi}(\eta, q) .
$$

Specifying this function, one then takes as an initial condition, $F\left(\eta_{\text {init }}, q\right)$, a realization of a normal distribution with zero mean and variance given by Eq. (8), and some suitable condition for the initial first derivative. With these initial conditions, one solves for (4), so one can now compute the potential (3). Topology is considered by restricting in (3) to normalized solutions of the Helmholtz equation satisfying the invariance conditions (6). This method has been extensively used in $[5,12,13]$, although in the former the authors do not consider the randomness of the function $F(\eta, q)$. Instead the random character of the CMB maps is attributed exclusively to the random character of the eigenmodes of the Laplacian in compact hyperbolic spaces.

The second method to produce simulated maps of CMB temperature anisotropies in universes with nontrivial spatial topology was first described in [14], and used in $[15,16]$. It is based in considering the randomness of the Newtonian potential in the eigenmodes of the Laplacian operator. We begin by decomposing the general solution of the Helmholtz equation, in the universal covering space, as a sum of fundamental solutions

$$
\xi(q, \mathbf{x})=\sum_{\ell, m} \hat{\xi}_{\ell m}(q) \mathcal{Y}_{\ell m}(q, \mathbf{x}),
$$

where

$$
\mathcal{Y}_{\ell m}(q, \mathbf{x})=\rho_{\ell}(q, x) Y_{\ell m}(\mathbf{n})
$$

is the normalized solution of the Helmholtz equation after separating it in radial and angular variables. Here we have put $x=|\mathbf{x}|, \mathbf{n}$ is the unit vector in the direction of $\mathbf{x}$, and the $Y_{\ell m}(\mathbf{n})$ are the spherical harmonic functions (see Appendix A). Since the solutions (10) are normalized, the randomness of the eigenmodes' amplitudes relies on the coefficients $\hat{\xi}_{\ell m}(q)$.

Introducing (3) in (2), and using (9) and (10), we arrive at the decomposition of the temperature anisotropy map in spherical harmonics

$$
\frac{\delta T}{T}(\mathbf{n})=\sum_{\ell, m} a_{\ell m} Y_{\ell m}(\mathbf{n}),
$$

with multipole coefficients

$$
a_{\ell m}=\int d q \hat{\xi}_{\ell m} s(q) G_{\ell}(q),
$$


and the effects of physical cosmology given by

$$
\begin{aligned}
G_{\ell}(q)= & \frac{1}{3} F\left(\eta_{\mathrm{LSS}}, q\right) \rho_{\ell}\left(q, R_{\mathrm{LSS}}\right) \\
& +\left.2 \int_{\eta_{\mathrm{LSS}}}^{\eta_{0}} d \eta \frac{\partial F}{\partial \eta}\right|_{(\eta, q)} \rho_{\ell}(q, R(\eta)) .
\end{aligned}
$$

At this point it is convenient to recall how topology enters in the story. Note that, due to the invariance conditions (6), not every solution of the form (9) is a solution in a quotient space. However, one would expect that any solution in a quotient space could be written in this form, the only problem being to find the correct coefficients $\hat{\xi}_{\ell m}(q)$. These coefficients are not independent one from the other, since the invariance conditions (6) establish certain relations among them. In what follows we will assume that these relations can always be found, so that we will always represent an eigenmode in a quotient space by Eq. (9), with suitable coefficients.

A crucial point in [14] is the decomposition of these coefficients as

$$
\hat{\xi}_{\ell m}(q)=\sqrt{P_{\Phi}(q)} \hat{\mathbf{e}}_{\ell m}(q),
$$

where $P_{\Phi}(q)$ is the gravitational initial power spectrum, and the $\hat{\mathbf{e}}_{\ell m}(q)$ form a multivariate Gaussian random variable, with a nondiagonal covariance matrix due to the relations among the coefficients $\hat{\xi}_{\ell m}(q)$ coming from the invariance conditions.

The simulation procedure can now be described. First we solve Eq. (4) using the initial condition $F\left(\eta_{\text {init }}, q\right)=1$, and a suitable condition for the first derivative, and use this in (13) to compute $G_{\ell}(q)$. Then generate a realization of the random variable $\hat{\mathbf{e}}_{\ell m}(q)$ and use (14) to compute $\hat{\xi}_{\ell m}(q)$. The map is now simulated by computing the coefficients $a_{\ell m}$ using (12), and performing the sum in (11).

An alternative method of simulation, also proposed in [14], is to construct the covariance matrix of the $a_{\ell m}$ 's as

$$
\left\langle a_{\ell m} a_{\ell^{\prime} m^{\prime}}^{*}\right\rangle=\int d q d q^{\prime} G_{\ell}(q) G_{\ell^{\prime}}\left(q^{\prime}\right)\left\langle\hat{\xi}_{\ell m}(q) \hat{\xi}_{\ell^{\prime} m^{\prime}}^{*}\left(q^{\prime}\right)\right\rangle .
$$

The substitution of (14) into (15), and the evaluation of the resulting integral give rise to expressions for the covariance matrix in terms of the eigenvalues and eigenmodes of the Laplacian operator. The multipolar coefficients are then obtained directly as a realization of a Gaussian distribution with zero mean and covariance given by (15).

The method we propose in this paper lies along these lines, but we are able to manipulate the correlation function for the $\hat{\xi}_{\ell m}(q)$ in a way that avoids the need for an explicit determination of the eigenmodes of the Laplacian. Instead, the final expression after the integration of (15) is given in terms of the isometries of the corresponding covering group.
Our starting point is an expression, obtained by Bond $e t$ $a l$. in [17], that relates the two-point correlation function of the Newtonian potential in a simply connected universe, and that in a multiply connected universe, when both potentials have the same initial power spectrum. For a homogeneous and isotropic random field the expression is

$$
\left\langle\Phi(\eta, \mathbf{x}) \Phi\left(\eta, \mathbf{x}^{\prime}\right)\right\rangle^{\Gamma}=\sum_{g \in \Gamma}|g|\left\langle\Phi(\eta, \mathbf{x}) \Phi\left(\eta, g \mathbf{x}^{\prime}\right)\right\rangle^{\text {s.c. }},
$$

where $|g|=1$ if $g$ is orientation preserving, and -1 otherwise.

We now show how to use Eq. (16) in order to express (15) in terms of the covering group, and for simplicity we will restrict the presentation to flat topologies. In Euclidean space, the most general solution of Eq. (5) is written in the form

$$
\xi(q, \mathbf{x})=\int d^{3} k \delta(q-k) \hat{\xi}(\mathbf{k}) e^{i \mathbf{k} \cdot \mathbf{x}} .
$$

If we now expand the plane waves in spherical harmonics as

$$
e^{i \mathbf{k} \cdot \mathbf{x}}=4 \pi \sum_{\ell, m} i^{\ell} j_{\ell}(k x) Y_{\ell m}^{*}\left(\mathbf{n}_{\mathbf{k}}\right) Y_{\ell m}(\mathbf{n}),
$$

where $j_{\ell}(x)$ is the spherical Bessel function of order $\ell$, and introduce it in (17) we obtain $\xi(q, \mathbf{x})$ expressed as in Eq. (9) with

$$
\hat{\xi}_{\ell m}(q)=4 \pi i^{\ell} \int d^{3} k \delta(q-k) \hat{\xi}(\mathbf{k}) Y_{\ell m}^{*}\left(\mathbf{n}_{\mathbf{k}}\right),
$$

where $\mathbf{n}_{\mathbf{k}}$ is the unit vector in the direction of $\mathbf{k}$, and $\rho_{\ell}(q, x)=j_{\ell}(q x)$.

Note that we have not decomposed $\hat{\xi}_{\ell m}(q)$ as in Eq. (14). Instead, the decomposition (19) allows us to implement the randomness of the Newtonian potential in the modes $\hat{\xi}(\mathbf{k})$. In fact, introducing (19) in (15), the covariance matrix for the $a_{\ell m}$ 's now reads

$$
\begin{aligned}
\left\langle a_{\ell m} a_{\ell^{\prime} m^{\prime}}^{*}\right\rangle= & (4 \pi)^{2} i^{\ell-\ell^{\prime}} \int d^{3} k d^{3} k^{\prime} G_{\ell}(k) G_{\ell^{\prime}}\left(k^{\prime}\right) \\
& \times\left\langle\hat{\xi}(\mathbf{k}) \hat{\xi}^{*}\left(\mathbf{k}^{\prime}\right)\right\rangle Y_{\ell m}^{*}\left(\mathbf{n}_{\mathbf{k}}\right) Y_{\ell^{\prime} m^{\prime}}\left(\mathbf{n}_{\mathbf{k}^{\prime}}\right) .
\end{aligned}
$$

It is the correlation function of the modes $\hat{\xi}(\mathbf{k})$ that carries all the topological information, as we will see in the following.

Introducing (17) in (3) we obtain

$$
\Phi(\eta, \mathbf{x})=\int d^{3} k F(\eta, k) \hat{\xi}(\mathbf{k}) e^{i \mathbf{k} \cdot \mathbf{x}},
$$

thus the two-point correlation function of the Newtonian potential now reads

$$
\begin{aligned}
\left\langle\Phi(\eta, \mathbf{x}) \Phi\left(\eta, \mathbf{x}^{\prime}\right)\right\rangle= & \int d^{3} k d^{3} k^{\prime} F(\eta, k) F\left(\eta, k^{\prime}\right) \\
& \times\left\langle\hat{\xi}(\mathbf{k}) \hat{\xi}^{*}\left(\mathbf{k}^{\prime}\right)\right\rangle e^{i\left(\mathbf{k} \cdot \mathbf{x}-\mathbf{k}^{\prime} \cdot \mathbf{x}^{\prime}\right)} .
\end{aligned}
$$


At this point we have to recall that a Euclidean isometry can always be written as $g=(R, \mathbf{r})$, where $R$ is an orthogonal transformation and $\mathbf{r}$ is an Euclidean vector, and that this isometry acts on a vector $\mathbf{x}$ as $g \mathbf{x}=R \mathbf{x}+\mathbf{r}$. It is now an easy task to deduce from Eqs. (16) and (21) that

$$
\left\langle\hat{\xi}(\mathbf{k}) \hat{\xi}^{*}\left(\mathbf{k}^{\prime}\right)\right\rangle^{\Gamma}=\sum_{g \in \Gamma}\left\langle\hat{\xi}(\mathbf{k}) \hat{\xi}^{*}\left(R \mathbf{k}^{\prime}\right)\right\rangle^{\text {s.c. }} e^{-i R \mathbf{k}^{\prime} \cdot \mathbf{r}} .
$$

In most inflationary models the initial perturbations of the gravitational field are homogeneous and isotropic Gaussian random fields, thus the correlation matrix of the $\mathbf{k}$ modes in a simply connected universe takes the form

$$
\left\langle\hat{\xi}(\mathbf{k}) \hat{\xi}^{*}\left(\mathbf{k}^{\prime}\right)\right\rangle^{\text {s.c. }}=\frac{P_{\Phi}(k)}{k^{3}} \delta\left(\mathbf{k}-\mathbf{k}^{\prime}\right) .
$$

The use of (22) now yields

$$
\left\langle\hat{\xi}(\mathbf{k}) \hat{\xi}^{*}\left(\mathbf{k}^{\prime}\right)\right\rangle^{\Gamma}=\frac{P_{\Phi}(k)}{k^{3}} \sum_{g \in \Gamma} \delta\left(\mathbf{k}-R \mathbf{k}^{\prime}\right) e^{-i R \mathbf{k}^{\prime} \cdot \mathbf{r}},
$$

for the correlation matrix of the $\mathbf{k}$ modes in the quotient space $M=\tilde{M} / \Gamma$, which when substituted in (20) finally gives

$$
\left\langle a_{\ell m} a_{\ell^{\prime} m^{\prime}}^{*}\right\rangle^{\Gamma}=(4 \pi)^{2} i^{\ell-\ell^{\prime}} \int \frac{d^{3} k}{k^{3}} \Psi_{\ell \ell^{\prime}}(k) Y_{\ell^{\prime} m^{\prime}}^{\Gamma}(\mathbf{k}) Y_{\ell m}^{*}\left(\mathbf{n}_{\mathbf{k}}\right),
$$

where the physical effects are encoded in

$$
\Psi_{\ell \ell^{\prime}}(k)=P_{\Phi}(k) G_{\ell}(k) G_{\ell^{\prime}}(k),
$$

and the topological information in

$$
\Upsilon_{\ell m}^{\Gamma}(\mathbf{k})=\sum_{g \in \Gamma} e^{-i \mathbf{k} \cdot \mathbf{r}} Y_{\ell m}\left(\mathbf{n}_{R^{T} \mathbf{k}}\right) .
$$

The integration in (23) is over the whole $\mathbf{k}$ space. The topological information is carried in Eq. (25), which automatically selects the eigenvalues of the Laplacian operator in $M$. This can be seen in Appendix D, where $Y_{\ell m}^{\Gamma}(\mathbf{k})$ is expressed in terms of Dirac's delta functions centered in the eigenvalues of the Laplacian operator in the corresponding quotient spaces.

\section{DECOMPOSITION OF $\Gamma$ IN CYCLIC SUBGROUPS}

In this section we develop some formal results in order to proceed further. Especially, we define the topological signature of any covariance function that can be decomposed as

$$
X^{\Gamma}=\sum_{g \in \Gamma} X^{g},
$$

as, for example, the two-point correlation function of the Newtonian potential and the correlation matrix $\left\langle a_{\ell m} a_{\ell^{\prime} m^{\prime}}^{*}\right\rangle$. Then we work out a suitable decomposition of a covering group in their cyclic subgroups, and write down the topo- logical signature in terms of this decomposition. We also show here that the symmetries of a quotient space appear transparently in the decomposition of its covering group in cyclic subgroups. It follows that the main result of this section is the elucidation of how these symmetries manifest themselves in the topological signature of CMB temperature anisotropy maps.

Let us begin by writing the obvious decomposition

$$
X^{\Gamma}=X^{\text {s.c. }}+X^{\hat{\Gamma}},
$$

where $\hat{\Gamma}=\Gamma \backslash\{i d\}$. The second term in the right-hand side is the topological signature in the covariance function. The expressions we present in the following are analogous to (26) and are also rather obvious.

It is convenient to introduce a notation, so natural, that has been used in (26) without any previous definition. Let $S$ be any subset of isometries of the covering space $\tilde{M}$, then a superscript $S$ in the covariance function means

$$
X^{S}=\sum_{g \in S} X^{g} .
$$

Then if $M=\tilde{M} / \Gamma$ is a quotient space and $\Gamma_{1} \subset \Gamma$ is any subset of the covering group, we can immediately write $X^{\Gamma}=X^{\Gamma_{1}}+X^{\Gamma \backslash \Gamma_{1}}$. This expression is nothing but the simplest generalization of Eq. (26), which corresponds to the trivial case $\Gamma_{1}=\{i d\}$. We get a further generalization as follows: let $\Gamma_{1}$ and $\Gamma_{2}$ be any two subsets of the covering group $\Gamma$, such that $\Gamma_{1} \cap \Gamma_{2}=\Gamma_{3}$, then

$$
X^{\Gamma}=X^{\Gamma_{1}}+X^{\Gamma_{2}}-X^{\Gamma_{3}}+X^{\Gamma \backslash\left(\Gamma_{1} \cup \Gamma_{2}\right)} .
$$

We can now write the formal result we are interested in. Consider the subsets $G_{1}, \ldots, G_{n} \subset \Gamma$ such that for any $i \neq$ $j, G_{i} \cap G_{j}=H$, then by induction on (27) we get

$$
X^{\Gamma}=\sum_{i=1}^{n} X^{G_{i}}-(n-1) X^{H}+X^{\Gamma \backslash G},
$$

where $G=\cup G_{i}$.

To move forward, let $G_{1}=\left\langle g_{1}\right\rangle$ and $G_{2}=\left\langle g_{2}\right\rangle$ be two cyclic subgroups of $\Gamma$, and let $\mathbf{0} \in \tilde{M}$ be a lift to $\tilde{M}$ of the position of the observer in $M$. We will say that $g_{1}$ and $g_{2}$ are conjugate by an isometry that "does not move the observer" if there exists an isometry $\eta$ fixing $\mathbf{0}$ and such that $g_{1}=\eta^{-1} g_{2} \eta$. Note that, as a consequence, we have that $d\left(\mathbf{0}, g_{1} \mathbf{0}\right)=d\left(\mathbf{0}, g_{2} \mathbf{0}\right)$, where $d(\mathbf{x}, \mathbf{y})$ is the distance between two points $\mathbf{x}$ and $\mathbf{y}$ in $\tilde{M}$. By extension, we will also say that the groups $G_{1}$ and $G_{2}$ are conjugate by an isometry that does not move the observer. In addition, we will say that $g_{1}$ is a minimal distance generator of $G_{1}$ (with respect to the observer) if $d\left(\mathbf{0}, g_{1} \mathbf{0}\right) \leq d(\mathbf{0}, \gamma \mathbf{0})$ for any other generator $\gamma \in G_{1}$.

Now consider the isometries $g_{1}, \ldots, g_{n} \in \Gamma$ that generate the cyclic groups $G_{i}=\left\langle g_{i}\right\rangle$, and such that if $i \neq j$ then $G_{i} \cap G_{j}=\{i d\}$. By using Eq. (28) we immediately obtain that the topological signature of the covariance function 
can be decomposed as

$$
X^{\hat{\Gamma}}=\sum_{i=1}^{n} X^{\hat{G}_{i}}+X^{\Gamma \backslash G} .
$$

In the following we will be particularly interested in the case where the $g_{i}$ 's are minimal distance generators of the $G_{i}$ 's, and the latter form a complete set of groups mutually conjugate by isometries that do not move the observer.

Decomposing the topological signature further along these lines, let $g_{1}, \ldots, g_{n}, h_{1}, \ldots, h_{m} \in \Gamma$ be minimal distance generators of the groups $G_{i}=\left\langle g_{i}\right\rangle$ and $H_{j}=\left\langle h_{j}\right\rangle$, and let $G=\cup G_{i}$ and $H=\cup H_{j}$. Moreover, suppose that the $G_{i}$ 's and the $H_{j}$ 's form two complete sets of groups mutually conjugate by isometries that do not move the observer, and such that $G \cap H=\{i d\}$ and $d\left(\mathbf{0}, g_{1} \mathbf{0}\right) \leq$ $d\left(\mathbf{0}, h_{1} \mathbf{0}\right)$. Then the topological signature can be decomposed as

$$
X^{\hat{\Gamma}}=\sum_{i=1}^{n} X^{\hat{G}_{i}}+\sum_{i=1}^{m} X^{\hat{H}_{i}}+X^{\Gamma \backslash(G \cup H)} .
$$

We can proceed along these lines again and again, and obtain the following decomposition, in cyclic subgroups, of the covering group $\Gamma$,

$$
\Gamma=\bigcup_{i=1}^{\infty} \bigcup_{j=1}^{k_{i}} \Gamma_{i j},
$$

where $g_{i j} \in \Gamma$ is a minimal distance generator of the cyclic group $\Gamma_{i j}$, and such that

(1) For each $i \in \mathbb{N}$, the set $\left\{\Gamma_{i 1}, \ldots, \Gamma_{i k_{i}}\right\}$ is a complete set of groups mutually conjugate by isometries that do not move the observer.

(2) If $i \neq i^{\prime}$, the sets $\cup_{j=1}^{k_{i}} \Gamma_{i j}$ and $\cup_{j=1}^{k_{i^{\prime}}} \Gamma_{i^{\prime} j}$ have the identity as the only common element.

(3) If $i<i^{\prime}$, then $d\left(\mathbf{0}, g_{i 1} \mathbf{0}\right) \leq d\left(\mathbf{0}, g_{i^{\prime} 1} \mathbf{0}\right)$.

Then the topological signature of the covariance function can be written as

$$
X^{\hat{\Gamma}}=\sum_{i=1}^{\infty} \sum_{j=1}^{k_{i}} X^{\hat{\Gamma}_{i j}}
$$

Thus, to compute the topological signature of the covariance function for any multiply connected manifold, it is enough to know how to compute it for manifolds whose covering groups are cyclic groups. It is now obvious that this decomposition will be particularly useful for calculating the correlation matrix of the $a_{\ell m}$ 's for any compact manifold once we know how to calculate it for cyclic flat (twisted cylinders), spherical (lens spaces), and hyperbolic manifolds.

Let us now show that the decomposition (29) describes transparently the symmetries of the quotient manifold $M=\tilde{M} / \Gamma$. Actually, this decomposition contains the symmetries of the Dirichlet fundamental polyhedron of $M$ centered at the observer's position $\mathbf{0} \in \tilde{M}$, which is what one expects to reconstruct with cosmological observations. Recall that the Dirichlet fundamental polyhedron centered at $\mathbf{0} \in \tilde{M}$ is the set $\mathcal{D}_{\mathbf{0}} \subset \tilde{M}$ defined by (see [22])

$$
\mathcal{D}_{\mathbf{0}}=\{\mathbf{x} \in \tilde{M}: d(\mathbf{0}, \mathbf{x}) \leq d(g \mathbf{0}, \mathbf{x}) \text { for any } g \in \Gamma\} .
$$

The first thing to be noted is that, although the whole covering group enters in this definition, it turns out that, in order to effectively construct the Dirichlet polyhedron, we only need the minimal distance generators (and maybe the first few positive powers) of the first few cyclic groups $\Gamma_{i j}$ and their inverses.

In fact, for each $g \in \Gamma$ consider the semispace

$$
H_{g}=\{\mathbf{x} \in \tilde{M}: d(\mathbf{0}, \mathbf{x}) \leq d(g \mathbf{0}, \mathbf{x})\} .
$$

Then it is obvious that the Dirichlet polyhedron is the intersection of all of these semispaces. However, there is a high redundancy here since, for a sufficiently large positive power $n$, we may have

$$
\bigcap_{k=1}^{n-1} H_{g_{i j}^{k}} \subset H_{g_{i j}^{n}}
$$

and so this and further powers of $g_{i j}$ do not effectively contribute to the polyhedron $\mathcal{D}_{\mathbf{0}}$. Additionally, if some $H_{g}$ effectively contributes to the polyhedron, so does $H_{g^{-1}}$, thus the same argument holds for the inverses of the minimal distance generators. Moreover, note that due to condition 3 above, for a sufficiently large $i$, it may be the case that the semispaces $H_{g_{i j}}$ do not contribute effectively to the polyhedron.

The faces of the Dirichlet polyhedron are subsets of the boundary planes of the semispaces effectively contributing to it. In fact, for each $H_{g}$ effectively contributing, the corresponding face is orthogonal to the geodesic joining $\mathbf{0}$ and $g \mathbf{0}$, and cuts it at its middle point. It follows that the decomposition (29) describes the symmetries of the Dirichlet fundamental polyhedron of $M$ centered at the observer.

\section{FLAT HOMOGENEOUS MANIFOLDS}

We have seen in the previous section that, to compute the topological signature of CMB temperature anisotropies in a given manifold, we just need to know how to compute it for the cyclic manifolds that cover it maximally. In the flat orientable case, the cyclic manifolds are twisted cylinders, i.e., manifolds with a covering group generated by a screw motion. We will now focus on the simplest case, the flat homogeneous manifolds, which are generated by translations only.

The flat homogeneous manifolds are 3-tori or $T^{3}$ manifolds (generated by three linearly independent translations), chimneys or $T^{2}$ manifolds (generated by two linearly independent translations), and cylinders or $T^{1}$ manifolds (generated by one translation). Thus, we first 
compute the correlation matrix $\left\langle a_{\ell m} a_{\ell^{\prime} m^{\prime}}^{*}\right\rangle^{\Gamma}$ for cylinders, and then show how the decomposition (30) is used to compute the topological signature in this matrix for twoand three-dimensional tori. We also show that the computation of the angular power spectrum in tori is greatly simplified by this decomposition.

\section{A. The cylinder}

Let us consider a cylinder orthogonal to the $z$ direction, that is with covering group generated by the translation $g=(I, \mathbf{a})$, with $\mathbf{a}=L \hat{\mathbf{e}}_{z}$, where distances are measured in units of the radius of the last scattering surface $R_{\mathrm{LSS}}$. This choice of the coordinate system is very convenient since here the cylinder appears invariant under (i) arbitrary rotations around the $z$ axis, (ii) the parity transformation, and (iii) the reflection on the $y=0$ plane. Thus, according to Sec. A 3, we will end with a real correlation matrix with no $m$-dependent correlations and the multipoles $\ell$ and $\ell^{\prime}$ correlated only when both are even or odd.

It is convenient to recall here that our cylinder has injectivity radius equal to $L / 2$, thus cylinders with $L<2$ are "small" and might present topological copies of discrete sources and/or circles in the sky. On the other hand, "large" cylinders, i.e., those with $L>2$, have undetectable topology with the methods currently available [23].

The covering group of the cylinder is labeled by the integers as $g^{n}=(I, n \mathbf{a})$, with $n \in \mathbb{Z}$. We then have from (25) that all the topological information is encoded in

$$
Y_{\ell m}^{\Gamma}(\mathbf{k})=\sum_{n \in \mathbb{Z}} e^{-i n k_{z} L} Y_{\ell m}\left(\mathbf{n}_{\mathbf{k}}\right),
$$

and thus the correlation matrix of the $a_{\ell m}$ 's for the cylinder is simply

$$
\begin{aligned}
\left\langle a_{\ell m} a_{\ell^{\prime} m^{\prime}}^{*}\right\rangle^{\Gamma}= & (4 \pi)^{2} i^{\ell-\ell^{\prime}} \int \frac{d^{3} k}{k^{3}} \Psi_{\ell \ell^{\prime}}(k) \\
& \times\left(\sum_{n \in \mathbb{Z}} e^{-i n k_{z} L}\right) Y_{\ell^{\prime} m^{\prime}}\left(\mathbf{n}_{\mathbf{k}}\right) Y_{\ell m}^{*}\left(\mathbf{n}_{\mathbf{k}}\right) .
\end{aligned}
$$

To reduce this integral we may use any of the following two identities, either

$$
\sum_{n \in \mathbb{Z}} e^{-i n k_{z} L}=2 \pi \sum_{p \in \mathbb{Z}} \delta\left(k_{z} L-2 \pi p\right),
$$

or

$$
\sum_{n \in \mathbb{Z}} e^{-i n k_{z} L}=1+2 \sum_{n=1}^{\infty} \cos \left(n k_{z} L\right) .
$$

The first identity is obvious since the left-hand side is the Fourier expansion of the right hand side. This option yields a formula of the kind obtained in Appendix D, which expresses the correlation matrix in terms of the eigenvalues of the Laplacian operator on the cylinder. The second one still uses a parametrization in terms of the covering group, and thus can be used to isolate the topological signature.
In fact, using (33) to evaluate (31), and integrating in spherical coordinates, we get

$$
\left\langle a_{\ell m} a_{\ell^{\prime} m^{\prime}}^{*}\right\rangle^{\Gamma}=\left\langle a_{\ell m} a_{\ell^{\prime} m^{\prime}}^{*}\right\rangle^{\text {s.c. }}+\left\langle a_{\ell m} a_{\ell^{\prime} m^{\prime}}^{*}\right\rangle^{\hat{\Gamma}},
$$

where the simply connected part is as usual

$$
\left\langle a_{\ell m} a_{\ell^{\prime} m^{\prime}}^{*}\right\rangle^{\text {s.c. }}=C_{\ell}^{\text {s.c. }} \delta_{\ell \ell^{\prime}} \delta_{m m^{\prime}},
$$

with the (simply connected) angular power spectrum given by

$$
C_{\ell}^{\text {s.c. }}=(4 \pi)^{2} \int_{0}^{\infty} \frac{d x}{x} \Psi_{\ell \ell}(x),
$$

and the topological signature for the correlation matrix is given by

$\left\langle a_{\ell m} a_{\ell^{\prime} m^{\prime}}^{*}\right\rangle^{\hat{\Gamma}}=(4 \pi)^{2} i^{\ell-\ell^{\prime}} \delta_{\ell \ell^{\prime}}^{\bmod (2)} \delta_{m m^{\prime}} \int_{0}^{\infty} \frac{d x}{x} \Psi_{\ell \ell^{\prime}}\left(\frac{x}{L}\right) F_{\ell \ell^{\prime}}^{m}(x)$,

with

$$
F_{\ell \ell^{\prime}}^{m}(x)=2 \sum_{n=1}^{\infty} \int_{-1}^{1} d y \cos (n x y) \mathcal{P}_{\ell}^{m}(y) \mathcal{P}_{\ell^{\prime}}^{m}(y),
$$

where $\mathcal{P}_{\ell}^{m}(x)$ is the normalized associated Legendre function (see Appendix A). As expected, we have ended up with a real correlation matrix with factors $\delta_{\ell \ell^{\prime}}^{\bmod (2)}$ and $\delta_{m m^{\prime}}$.

After evaluating the series in (38), it turns out that $F_{\ell \ell^{\prime}}^{m}(x)$ is a piecewise continuous function. In fact, in each interval $[2 \pi q, 2 \pi(q+1)]$, it is a polynomial of degree $\left(\ell+\ell^{\prime}+1\right)$ in $\pi / x$. Indeed, the final result is

$$
F_{\ell \ell^{\prime}}^{m}(x)=\sum_{q \in \mathbb{Z}} \mathcal{F}_{\ell \ell^{\prime}}^{m}(x, q) \Theta(x-2 \pi q) \Theta(2 \pi(q+1)-x),
$$

where $\Theta(x)$ is the Heaviside step function, and the form of the polynomial $\mathcal{F}_{\ell \ell^{\prime}}^{m}(x, q)$ in the $q$ th interval of length $2 \pi$ is

$$
\begin{aligned}
\mathcal{F}_{\ell \ell^{\prime}}^{m}(x, q)= & 4 \sum_{k=0}^{\left(\ell+\ell^{\prime}\right) / 2}(-1)^{k} \mathcal{P}_{\ell \ell^{\prime} m}^{(2 k)}(0) g_{2 k+1}(q)\left(\frac{\pi}{x}\right)^{2 k+1} \\
& -\delta_{\ell \ell^{\prime}} .
\end{aligned}
$$

Here $g_{k}(q)$ are polynomials of degree $k$ in $q$, and $\mathcal{P}_{\ell \ell^{\prime} m}^{(k)}(0)$ is the $k$ th derivative of the polynomial

$$
\mathcal{P}_{\ell \ell^{\prime} m}(x)=\mathcal{P}_{\ell}^{m}(x) \mathcal{P}_{\ell^{\prime}}^{m}(x)
$$

evaluated at the origin. In Appendix B we present recurrence relations for the polynomials $g_{k}(q)$, and all the technical steps that take us from (38) to (39) can be found in Appendix C.

The integrals appearing in the topological signature (37) can be easily evaluated since Eqs. (39) and (40) allow an exact and very fast computation of the function $F_{\ell \ell^{\prime}}^{m}(x)$, and the integrands decay very fast, as illustrated in Figs. 1 and 2. In this figures we have adopted, for simplicity, a scale 

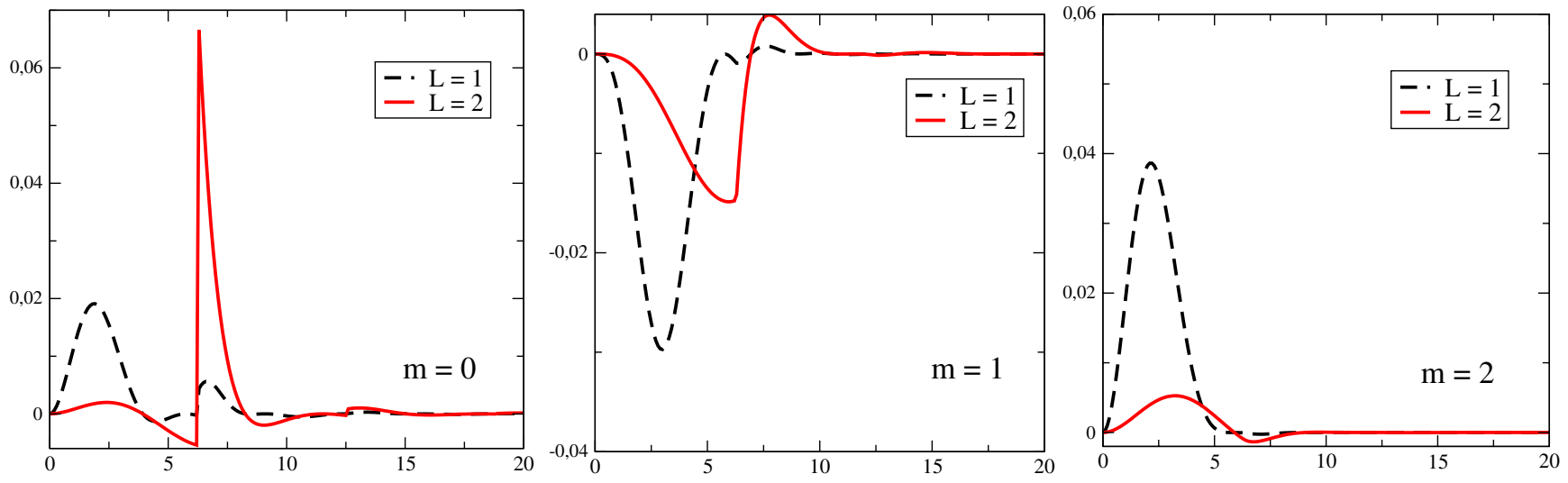

FIG. 1 (color online). Shapes of the integrands inside integrals of the type in (37) for $\ell=\ell^{\prime}=2$, and compactification scales $L=1$ and $L=2$ in units of the radius of the last scattering surface.

invariant Einstein-de Sitter model, thus

$$
\Psi_{\ell \ell^{\prime}}(x) \propto j_{\ell}(x) j_{\ell^{\prime}}(x)
$$

The nice behavior of the integrands in (37) is not a consequence of this particular choice of $\Psi_{\ell \ell^{\prime}}(x)$. Actually, the integrand in (37) always decays very fast because $\Psi_{\ell \ell^{\prime}}(x)$ and $F_{\ell \ell^{\prime}}^{m}(x)$ are both decaying functions, thus the evaluation of the topological signature for the cylinder is always very efficient.

The computation of the topological signature of the power spectrum reduces to a simple integral. In fact we obtain

$$
C_{\ell}^{\hat{\Gamma}}=(4 \pi)^{2} \int_{0}^{\infty} \frac{d x}{x} \Psi_{\ell \ell}\left(\frac{x}{L}\right) f_{\ell}(x),
$$

with

$$
f_{\ell}(x)=\frac{1}{2 \ell+1} \sum_{m=-l}^{\ell} F_{\ell \ell}^{m}(x) .
$$

Using (38) to perform this sum, the addition theorem for spherical harmonics (see Appendix A) yields immediately

$$
C_{\ell}^{\hat{\Gamma}}=2(4 \pi)^{2} \int_{0}^{\infty} \frac{d x}{x^{2}} \Psi_{\ell \ell}\left(\frac{x}{L}\right) \varphi_{1}(x),
$$
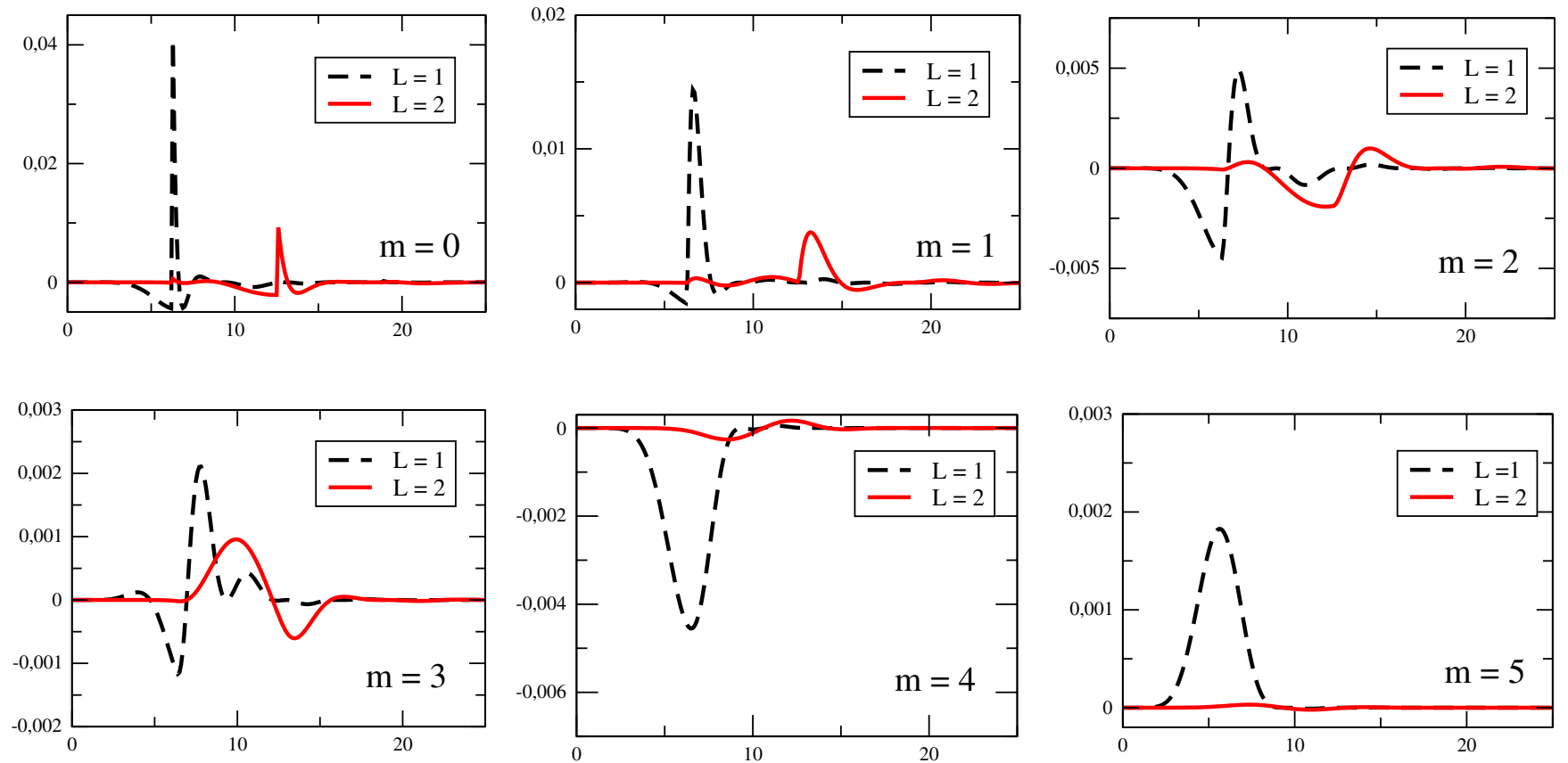

FIG. 2 (color online). Shapes of the integrands inside integrals of the type in (37) for $\ell=\ell^{\prime}=5$, and compactification scales $L=1$ and $L=2$ in units of the radius of the last scattering surface. 
where $\varphi_{1}(x)$ is the first Clausen $\varphi$-function given in Appendix B.

In Fig. 3(a) we show the low $\ell$ modes of the topological signature of the angular power spectrum of a cylinder, normalized with respect to (w.r.t.) $C_{\ell}^{\text {s.c. }}$, as a function of its size $L$. We can see that the topological signature is typically much smaller than the cosmic variance, even for small cylinders which have already been discarded observationally as candidates for the shape of our Universe because of the lack of antipodal matched circles in WMAP data [1,9]. Thus it is apparent that the angular power spectrum is not a good indicator to look for topology in this case.

The correlation matrix given by (34)-(40) corresponds to a cylinder for which the direction of compactification is parallel to the $z$ axis. The correlation matrix corresponding to a cylinder with a different orientation can be easily obtained from the previous one by simply rotating the celestial sphere. Thus, parametrizing the rotations with Euler angles, if $R(\alpha, \beta, \gamma) \in \mathrm{SO}(3)$ takes the $z$ axis to the direction of compactification of the cylinder, the topological signature of the corresponding correlation matrix can be computed using the expressions (A2)-(A4) of Appendix A yielding

$$
\left\langle a_{\ell m} a_{\ell^{\prime} m^{\prime}}^{*}\right\rangle_{R}^{\hat{\Gamma}}=e^{i\left(m^{\prime}-m\right) \alpha} \sum_{m_{1}} d_{m m_{1}}^{\ell}(\beta) d_{m^{\prime} m_{1}}^{\ell^{\prime}}(\beta)\left\langle a_{\ell m_{1}} a_{\ell^{\prime} m_{1}}^{*}\right\rangle^{\hat{\Gamma}},
$$

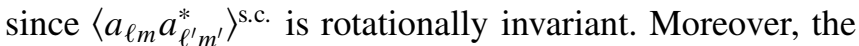
$\gamma$ angle does not appear in this expression since $R_{z}(\gamma)$ in (A3) does not move the $z$ axis, and $\left\langle a_{\ell m} a_{\ell^{\prime} m^{\prime}}^{*}\right\rangle^{\Gamma}$ is invariant under such rotations.

It should be noted here that, no matter its orientation, the cylinder is always invariant under parity, thus its correlation matrix will always conserve the factor $\delta_{\ell \ell^{\prime}}^{\bmod (2)}$. On the other hand, the correlation matrix will remain real as far as we perform rotations with $\alpha=0$, since in this case we do not rotate the cylinder around the $z$ axis, and thus it remains invariant under reflections on the plane $y=0$. However, any rotation of the cylinder (other than one with $\beta=\pi$ ) makes it noninvariant under azimuthal rotations; thus the correlation matrix of an arbitrarily oriented cylinder has $m$-dependent correlations. All these features can be seen explicitly in (44).

\section{B. Tori}

In order to calculate the correlation matrix of the $a_{\ell m}$ 's for a two- or a three-torus we use the decomposition (29) of its covering group in cyclic subgroups. Let $\Gamma_{i j}=\left\langle g_{i j}\right\rangle$ be the covering group of the cylinder generated by the element $g_{i j} \in \Gamma$, and let us write $L_{i}=d\left(\mathbf{0}, g_{i j} \mathbf{0}\right), g_{i}=$ $\left(I, L_{i} \hat{\mathbf{e}}_{z}\right)$, and $\Gamma_{i}=\left\langle g_{i}\right\rangle$. In the Euclidean case, the orientation preserving isometries that do not move the observer are rotations, thus let $R_{i j} \in \mathrm{SO}(3)$ be the rotation taking $\hat{\mathbf{e}}_{z}$ to the unit vector along $g_{i j} \mathbf{0}$.

Using the decomposition (30), we easily write the topological signature for the torus as a superposition of topological signatures of rotated cylinders. In fact,

$$
\left\langle a_{\ell m} a_{\ell^{\prime} m^{\prime}}^{*}\right\rangle^{\hat{\Gamma}}=\sum_{i=1}^{\infty} \sum_{j=1}^{k_{i}}\left\langle a_{\ell m} a_{\ell^{\prime} m^{\prime}}^{*}\right\rangle_{R_{i j}}^{\hat{\Gamma}_{i}},
$$

where the correlation matrices of the rotated cylinders are written in terms of the Wigner $D$ functions and Euler angles, according to (44), as

$$
\begin{aligned}
\left\langle a_{\ell m} a_{\ell^{\prime} m^{\prime}}^{*}\right\rangle_{R_{i j}}^{\hat{\Gamma}_{i}}= & e^{i\left(m^{\prime}-m\right) \alpha_{i j}} \sum_{m_{1}} d_{m m_{1}}^{\ell}\left(\beta_{i j}\right) d_{m^{\prime} m_{1}}^{\ell^{\prime}}\left(\beta_{i j}\right) \\
& \times\left\langle a_{\ell m_{1}} a_{\ell^{\prime} m_{1}}^{*}\right\rangle^{\hat{\Gamma}_{i}},
\end{aligned}
$$

and $\left(\beta_{i j}, \alpha_{i j}\right)$ are the angular spherical coordinates of the vector $g_{i j} \mathbf{0}$, and $k_{i}$ is the number of cylinders of size $L_{i}$. Since any group of translations is invariant under parity, from Sec. A 3 we know that the correlation matrix for a homogeneous flat manifold has always the factor $\delta_{\ell \ell^{\prime}}^{\bmod (2)}$, and this is evident from (45), since it is just a sum of correlation matrices of cylinders.

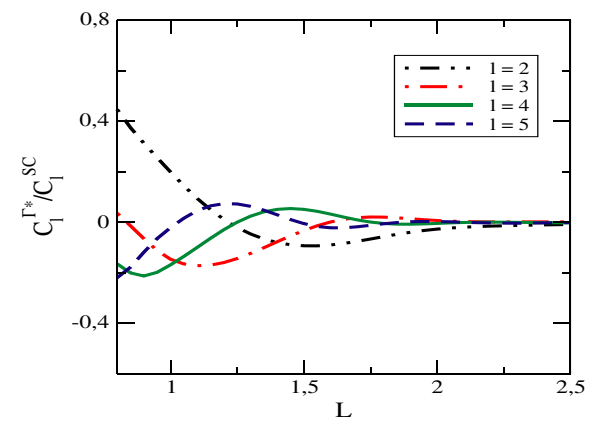

(a)

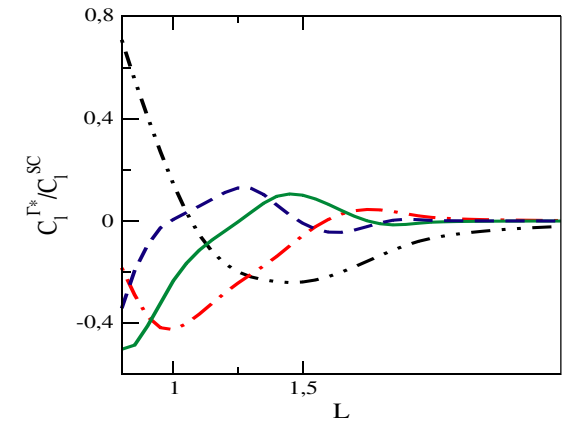

(b)

FIG. 3 (color online). Topological signature of the power spectrum of (a) a cylinder, and (b) a chimney with square base, for the first four $\ell$ modes, normalized w.r.t. $C_{\ell}^{\text {s.c. }}$, and as a function of the scale of compactification $L$. Note that, for each multipole, one can have a suppression or an excess of power depending on the value of $L$. For typographical reasons, here we write $\Gamma^{*}$ instead of $\hat{\Gamma}$. 
The power spectrum is rotationally invariant, thus from (45) one can easily write down the expression for the topological signature of the power spectrum of the torus as a superposition of topological signatures of power spectra of cylinders,

$$
C_{\ell}^{\hat{\Gamma}}=\sum_{i=1}^{\infty} k_{i} C_{\ell}^{\hat{\Gamma}_{i}}
$$

Let us consider a chimney with square base for the sake of illustration. It is convenient to orient the chimney so that its covering group consists of translations in the horizontal plane. We take as generators of the covering group the translations $g_{1}=(I, \mathbf{a})$ and $g_{2}=(I, \mathbf{b})$, with $\mathbf{a}=L \hat{\mathbf{e}}_{x}$ and $\mathbf{b}=L \hat{\mathbf{e}}_{y}$.

It is more convenient to reparametrize the cyclic decomposition as follows. Parametrize each cyclic subgroup by a pair of integer numbers $(p, q)$ as $G_{p q}=\left\langle g_{2}^{q} g_{1}^{p}\right\rangle$. Clearly, if the greatest common divisor of $(p, q)$ is $r$, then

$$
G_{p q}<G_{(p / r)(q / r)},
$$

where " $<$ " means "subgroup of." Thus we must restrict the labels to pairs $(p, q)$ of coprime numbers.

The only exceptions are when (i) $p= \pm 1$ and $q=0$ and vice versa, and (ii) when $p= \pm 1$ and $q= \pm 1$. Thus the first two complete sets of cyclic subgroups conjugate by a rotation are $\left\{G_{1,0}, G_{0,1}\right\}$ and $\left\{G_{1,1}, G_{-1,1}\right\}$. In both cases the conjugation is performed by a rotation of $\pi / 2$ around the $z$ axis. The compactification lengths of the corresponding cylinders are $L_{1,0}=L_{0,1}=L$ and $L_{1,1}=L_{-1,1}=\sqrt{2} L$, respectively. The Euler angles $(\beta, \alpha)$ to rotate the corresponding cylinders from the $z$ axis to their orientation in the chimney, according to (46), are $\beta=\pi / 2$ in all cases, and $\alpha_{1,0}=0, \quad \alpha_{0,1}=\pi / 2, \quad \alpha_{1,1}=\pi / 4$, and $\alpha_{-1,1}=$ $3 \pi / 4$, respectively.

To write the remaining complete sets of cyclic subgroups conjugate by a rotation let us define, for a pair of coprime natural numbers $(p, q)$, with $p>q \geq 1$, the groups

$$
\begin{array}{rlrl}
G_{p q}^{(1)} & =G_{p q}=\left\langle g_{2}^{q} g_{1}^{p}\right\rangle, & & G_{p q}^{(3)}=G_{-q, p}=\left\langle g_{2}^{p} g_{1}^{-q}\right\rangle, \\
G_{p q}^{(2)}=G_{q p}=\left\langle g_{2}^{p} g_{1}^{q}\right\rangle, & G_{p q}^{(4)}=G_{-p, q}=\left\langle g_{2}^{q} g_{1}^{-p}\right\rangle .
\end{array}
$$

The compactification lengths are all equal to $L_{p q}=$ $\sqrt{p^{2}+q^{2}} L$, and the Euler angles $(\beta, \alpha)$ to rotate the corresponding cylinders from the $z$ axis to their orientation in the chimney, according to (46), are $\beta=\pi / 2$ in all cases, and

$$
\begin{array}{ll}
\alpha_{p q}^{(1)}=\arctan \frac{q}{p}, & \alpha_{p q}^{(3)}=\frac{\pi}{2}+\alpha_{p q}^{(1)}, \\
\alpha_{p q}^{(2)}=\frac{\pi}{2}-\alpha_{p q}^{(1)}, & \alpha_{p q}^{(4)}=\pi-\alpha_{p q}^{(1)},
\end{array}
$$

respectively.
Let us denote by $\Gamma_{p q}$ the covering group of the cylinder with compactification scale $L_{p q}$ and oriented along the $z$ axis. Then, putting all this together, using (45) and (46), and taking into account the invariance properties derived in Sec. A 3, the topological signature of the chimney with square base is

$$
\left\langle a_{\ell m} a_{\ell^{\prime} m^{\prime}}^{*} \Gamma^{\hat{\Gamma}}=\delta_{m m^{\prime}}^{\bmod (4)} \sum_{m_{1}} d_{m m_{1}}^{\ell}(\pi / 2) d_{m^{\prime} m_{1}}^{\ell^{\prime}}(\pi / 2) \mathcal{W}_{\ell \ell^{\prime} m_{1}}^{m^{\prime}-m},\right.
$$

with

$$
\begin{aligned}
\mathcal{W}_{\ell \ell^{\prime} m_{1}}^{m}= & 2\left(\left\langle a_{\ell m_{1}} a_{\ell^{\prime} m_{1}}^{*}\right\rangle^{\hat{\Gamma}_{1,0}}+(-1)^{m / 4}\left\langle a_{\ell m_{1}} a_{\ell^{\prime} m_{1}}^{*}\right\rangle^{\hat{\Gamma}_{1,1}}\right) \\
& +4 \sum_{(p, q)} \cos m \alpha_{p q}^{(1)}\left\langle a_{\ell m_{1}} a_{\ell^{\prime} m_{1}}^{*}\right\rangle^{\hat{\Gamma}_{p q}},
\end{aligned}
$$

where the sum in $(p, q)$ is evaluated only for pairs of coprime natural numbers $(p, q)$ such that $p>q \geq 1$.

The topological signature of the power spectrum of the chimney with square base is simply

$$
C_{\ell}^{\hat{\Gamma}}=2\left(C_{\ell}^{\hat{\Gamma}_{1,0}}+C_{\ell}^{\hat{\Gamma}_{1,1}}\right)+4 \sum_{(p, q)} C_{\ell}^{\hat{\Gamma}_{p q}}
$$

Since the topological signature of the power spectrum of a cylinder converges quickly to zero as a function of the compactification scale [see Fig. 3(a)], it follows that the sum in (50) also converges quickly.

Moreover, the topological signature of the power spectrum of the chimney is larger than that of the cylinder. This is so because the $\ell$ th mode of the topological signature of the angular power spectrum of the cylinder oscillates very slowly. Thus, from (50) this signature is slightly higher in the chimney, as can be seen in Fig. 3(b). Actually, this is a general result that holds for manifolds whose covering groups are not cyclic.

\section{PATTERNS OF ALIGNMENT}

The nondiagonal character of the topological signature of the correlation matrix of the $a_{\ell m}$ 's in multiply connected universes and their $m$ dependence are manifestations of their globally anisotropic nature. They manifest themselves in statistically anisotropic temperature maps, i.e., realizations of random temperature fluctuations for which mean values of functions of the temperature over ensembles of universes depend on the orientation [10].

In this section we analyze an expected consequence of the topology of space on the temperature anisotropies of the CMB that has not received the deserved attention up to the present, namely, the existence of preferred directions in space. We show that the decomposition of the topological signature of the correlation matrix of the $a_{\ell m}$ 's in a universe with a complex topology, in signatures corresponding to cyclic topologies, demands the existence of "patterns of alignments" along these directions. For the sake of sim- 
plicity, we consider the Einstein-de Sitter model; thus from now on we will take (42) to perform all our calculations.

We want to call attention to the existence of alignments of the low $\ell$ modes of the CMB temperature maps in multiply connected universes. Indeed, in Fig. 4 we show a low resolution temperature map simulation for a cylinder with $L=2$ (in units of $R_{\mathrm{LSS}}$ ), together with the maps corresponding to the first four $\ell$ modes. One can see that these $\ell$ maps present alignments along the $z$ direction, which in this case is the unique direction of compactification of space.

Similar alignments as those present in our simulations have been reported as being observed in WMAP data, and have been attributed to a possible nontrivial topology of space with the shape of a cylinder [1]. These models have been quickly abandoned due to the lack of circles in the sky which should be present if the Universe were small [1,9]. However, our simulations show that even in universes slightly larger, and so not presenting such circles, these alignments should still be observable. Thus whether these observed alignments are a consequence of a nontrivial shape of our Universe is still an open question [24].

We will show here that, if our Universe had a nontrivial topology, its CMB temperature map will present characteristic patterns of alignment, even if its size is somewhat larger than the observable universe. Moreover, from the observed patterns of alignment, we might be able to reconstruct the shape of space.
In Fig. 5 we show the topological signature of the low $\ell$ modes of the diagonal part of the correlation matrix of the $a_{\ell m}$ 's, normalized w.r.t. $C_{\ell}^{\Gamma}$, for a cylinder oriented along the polar axis, as a function of the size $L$ of compactification. It is apparent that, for a given $\ell$ mode, there are multipole coefficients for which their expected values are above the mean (the angular power spectrum), and others for which these expected values are below it. This is the reason why the low $\ell$ modes in a cylinder are aligned. Actually, the expectation values $\left\langle\left|a_{\ell m}\right|^{2}\right\rangle$ are all equal to $C_{\ell}$ only in the simply connected case, thus the dispersion around the mean

$$
\sigma_{\ell}=\sqrt{\frac{1}{2 \ell+1} \sum_{m}\left(\left\langle\left|a_{\ell m}\right|^{2}\right\rangle-C_{\ell}\right)^{2}}
$$

is null. However, in a multiply connected universe, this dispersion is nonzero, and in a particular map, it adds to the cosmic variance. Thus it seems natural to propose the dispersion of the squares $\left|a_{\ell m}\right|^{2}$ around their mean value as a measure of these alignments in a map.

In Fig. 6 we show a plot of the dispersion (51), normalized w.r.t. $C_{\ell}^{\Gamma}$, for a cylinder oriented along the polar axis, as a function of $L$, for low multipoles. Note that even for a large cylinder $(L \approx 2)$ the dispersion is larger than $15 \%$ of the power for multipoles up to $\ell=5$. Indeed, on these scales the dispersion is of the order of the cosmic variance, and thus might be detectable.

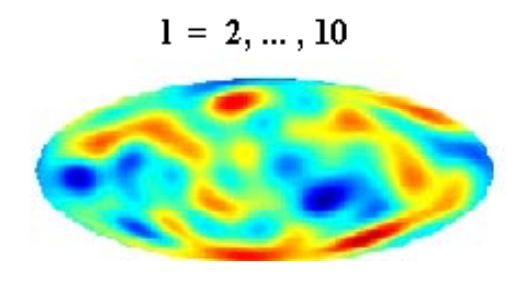

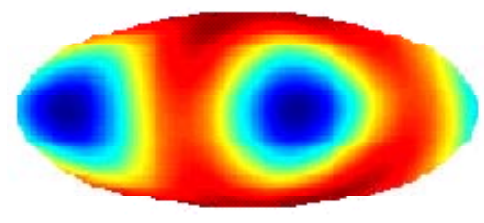

$1=2$

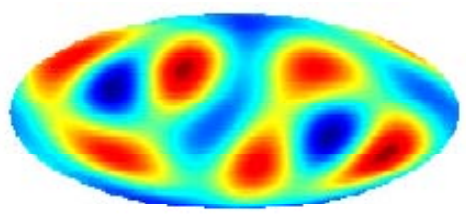

$1=4$

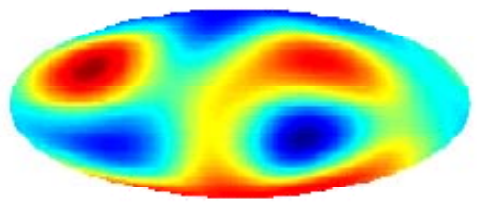

$1=3$

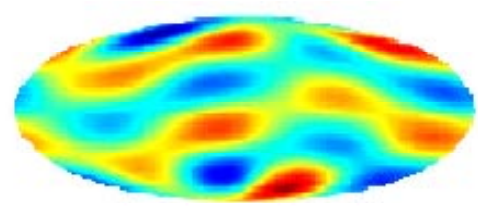

$1=5$

FIG. 4 (color online). Simulated CMB temperature anisotropy map at low resolution $(2 \leq \ell \leq 10)$ for a universe with a $T^{1}$ topology and size $L=2$. Also shown are the lowest multipoles, all of which present clear alignments near the polar direction. 

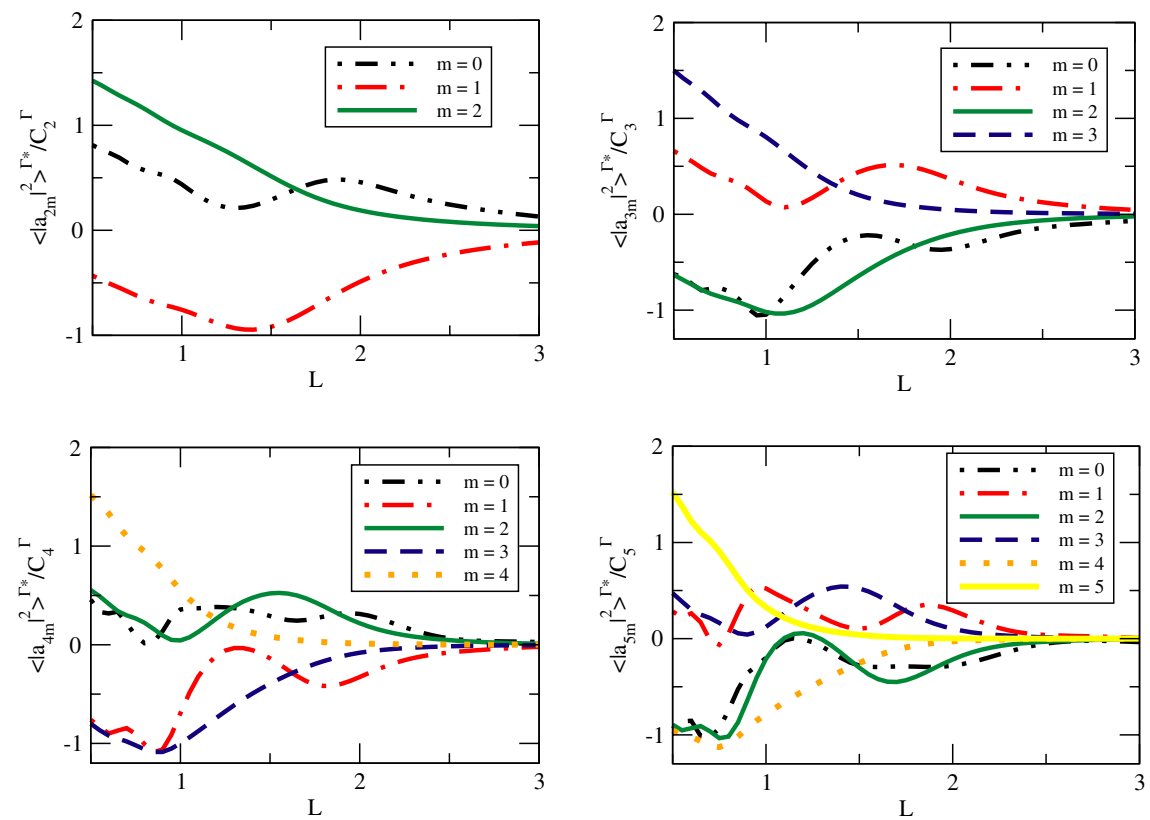

FIG. 5 (color online). Plots of the topological signatures of the squares $\left\langle\left|a_{\ell m}\right|^{2}\right\rangle^{\hat{\Gamma}}$, normalized w.r.t. $C_{\ell}^{\Gamma}$, for low multipoles $(2 \leq$ $\ell \leq 5$ ), in a universe with cylindrical topology aligned with the polar axis, and as a function of the scale of compactification $L$. For typographical reasons, here we write $\Gamma^{*}$ instead of $\hat{\Gamma}$.

In order to show that this is a good measure of the alignment of multipoles, and that it provides an efficient method to determine the directions of possible alignments in real or simulated maps, let us compute the dispersion of the squares $\left\langle\left|a_{\ell m}\right|^{2}\right\rangle$, Eq. (51), for a cylinder which is oriented along a direction making an angle $\beta$ with the $z$ axis. Each one of these squares can be computed with

$$
\left\langle\left|a_{\ell m}\right|^{2}\right\rangle_{R}^{\Gamma}=\sum_{m_{1}}\left[d_{m m_{1}}^{\ell}(\beta)\right]^{2}\left\langle\left|a_{\ell m_{1}}\right|^{2}\right\rangle^{\Gamma},
$$

which is nothing but (44) restricted to the diagonal part.

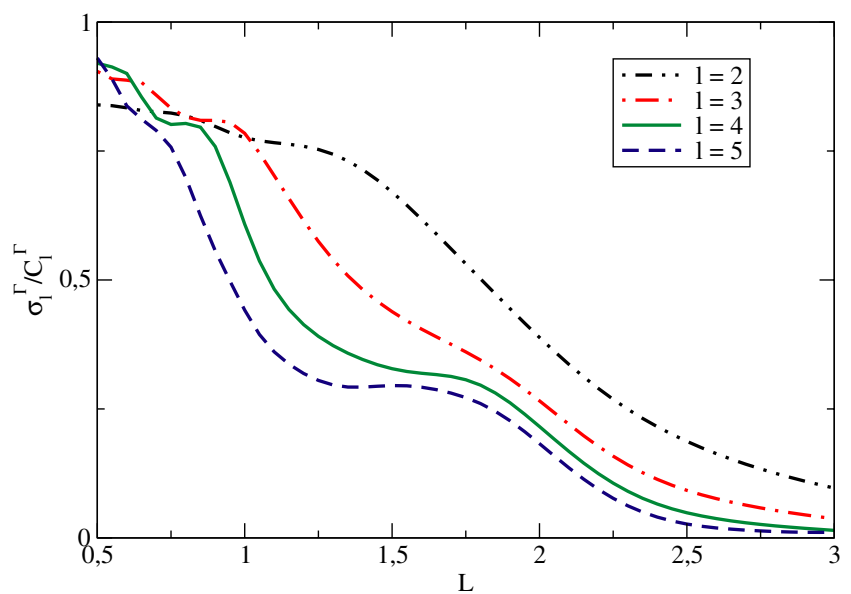

FIG. 6 (color online). Dispersion of the $\left\langle\left|a_{\ell m}\right|^{2}\right\rangle^{\Gamma}$, normalized w.r.t. $C_{\ell}^{\Gamma}$, for low multipoles $(2 \leq \ell \leq 5)$, in a universe with cylindrical topology aligned with the polar axis, and as a function of the scale of compactification $L$.
In Fig. 7 this dispersion is shown as a function of $\beta$ for different multipole coefficients and for different values of $L$. One can see that the dispersion has a maximum when the cylinder is oriented along the $z$ axis. Thus, in order to look for the alignments in a hypothetical universe with the shape of a cylinder, one should just rotate the celestial sphere around different directions until finding those two opposite ones along which the dispersion of the squares $\left|a_{\ell m}\right|^{2}$ is maximum.

However, in order to collect definitive evidence that the universe is indeed a cylinder, one should map the dispersion of the squares $\left|a_{\ell m}\right|^{2}$ on the sphere for each $\ell$ mode, i.e. one should determine the dispersion (51) as a function of the orientation of the celestial sphere. If the universe had the topology of a cylinder, these dispersion maps should be axially symmetric around a special direction, where the dispersion is maximum. Moreover, this direction should be identified with the direction of compactification of the cylinder.

If the universe has the topology of a flat homogeneous manifold, note from (45) and (46) that the topological signature is a superposition of rotated cylinders of different sizes. Thus, a CMB map for a universe with this kind of topology might present alignments along the directions corresponding to these cylinders. In fact, rotating the celestial sphere and computing the dispersion of the squares $\left|a_{\ell m}\right|^{2}$, an easy computation shows that, whenever we perform the rotation $R(0,-\theta,-\varphi)$ with $\theta=\beta_{i j}$ and $\varphi=$ $\alpha_{i j}$, one has the cylinder labeled by $(i, j)$ oriented along the polar axis, and thus dispersion maps might present local maxima along these directions. 

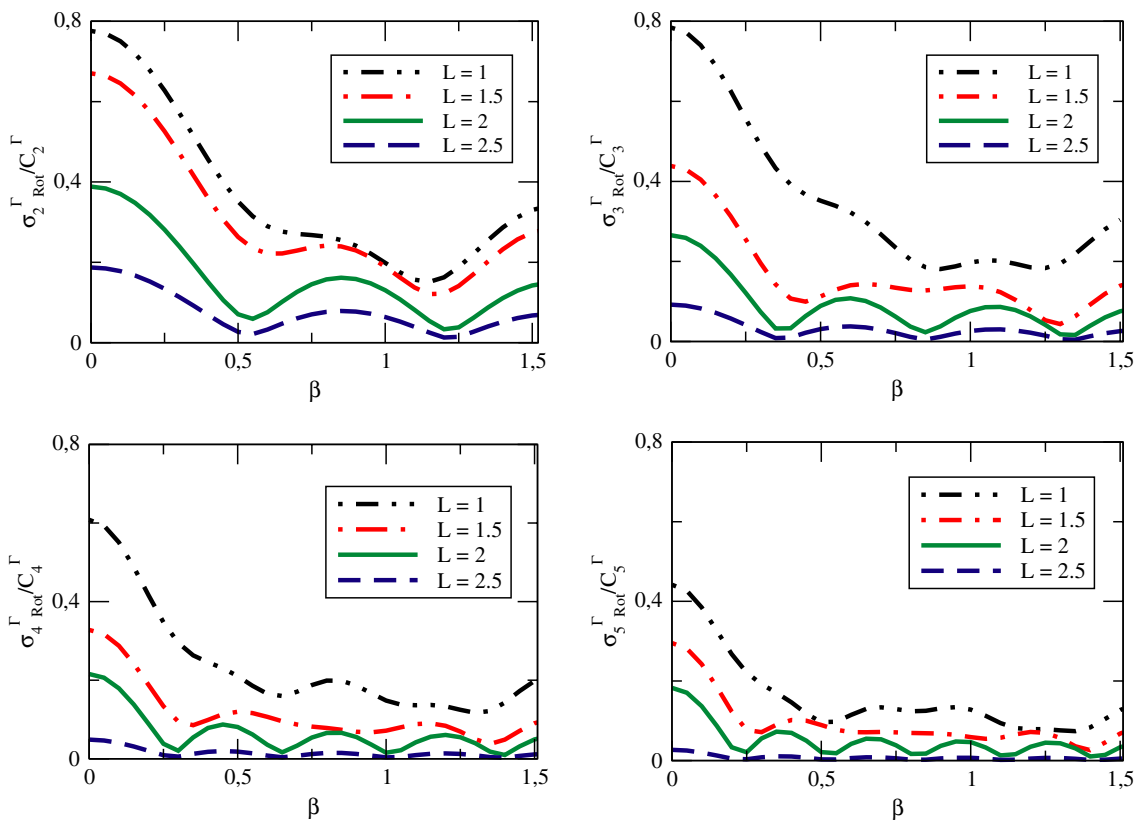

FIG. 7 (color online). Dispersion of the $\left\langle\left|a_{\ell m}\right|^{2}\right\rangle^{\Gamma}$, normalized w.r.t. $C_{\ell}^{\Gamma}$, for low multipoles $(2 \leq \ell \leq 5)$, in a universe with cylindrical topology, as a function of its orientation relative to the polar axis, and for different values of the scale of compactification $L$.

Whether these local maxima are observable in a given dispersion map will depend on (i) the scale of compactification of the corresponding cylinder $L_{i}$, (ii) the background due to the simply connected part, and (iii) the other cylinders' topological signatures. For large values of $L_{i}$, the corresponding local maxima will not be observable, however one can expect those maxima corresponding to the smaller cylinders to be detectable. The existence and distribution of these maxima in each dispersion map, together with their relative intensities is what we call a pattern of alignment.

It might seem that the problem of constructing dispersion maps for manifolds that are not flat homogeneous is more involved, since general cyclic manifolds do not have axial symmetry as the cylinder has. Equation (44) depends on two angles only because the cylinder is axially symmetric, but in the general case the expression for the correlation matrix in a rotated frame depends on the three Euler angles. Thus it seems at first sight that, in these cases, a dispersion map should be a function on the 3-sphere. Fortunately, the diagonal elements of the rotated correlation matrix depend only on the last two Euler angles as

$$
\left\langle\left|a_{\ell m}\right|^{2}\right\rangle_{R}^{\Gamma}=\sum_{m_{1}, m_{2}} e^{i\left(m_{2}-m_{1}\right) \gamma} d_{m m_{1}}^{\ell}(\beta) d_{m m_{2}}^{\ell}(\beta)\left\langle a_{\ell m_{1}} a_{\ell m_{2}}^{*}\right\rangle^{\Gamma},
$$

thus the same conclusion holds in the general case. Dispersion maps on the 2-sphere for low $\ell$ modes should display patterns of alignment showing the symmetries of our Universe if it has a (not too large) nontrivial topology.

\section{DISCUSSION}

In order to study systematically the effects of a nontrivial spatial topology in the temperature fluctuations of the $\mathrm{CMB}$, we need to have the ability to simulate efficiently temperature maps in multiply connected $\Lambda \mathrm{CDM}$ cosmologies. Almost all the usual methods to perform these simulations use explicitly the solutions of the Helmholtz equation in 3-manifolds with nontrivial topology. The computation of the eigenfunctions and eigenvalues of the Laplacian operator is simple only in Euclidean manifolds, while in spherical and hyperbolic spaces it is a nontrivial problem. In fact, it is only recently that an analytical computation has been achieved for all the spherical manifolds. The hyperbolic cases still have to be done numerically.

In this paper we have developed a simulation procedure that avoids the explicit use of the solutions of the Helmholtz equation. Instead, our results are expressed in terms of the covering group $\Gamma$ of the corresponding manifold. In this section we summarize the details of the method, its efficiency, the simple applications performed here, and discuss future related work.

\section{A. The formalism}

The cornerstone of our method is formula (16), which is the two-point correlation function of the scalar perturbations in a multiply connected universe expressed in terms of the covering group of the manifold [17]. By means of simple formal manipulations, we obtain an expression for the correlation matrix of the spherical harmonic expansion coefficients of the temperature maps, Eqs. (23)-(25), 
which contain all the topological information expressed as a sum over the elements of the covering group.

Former applications of (16) required a regularization procedure in order to account for divergences of the series, as well as some resummation techniques for accelerating the convergence. We do not have these problems here because the divergent series, which are actually distributions, appear only inside integrals. Indeed, on the one hand, we show in Appendix D that our formalism easily reproduces results previously reported in the literature, as well as some simple generalizations, without the need of any regularization procedure. On the other hand, elementary decompositions of the two-point correlation function (16), shown in Sec. III, guarantee that our final expressions are highly convergent, as discussed below.

Two decompositions of a generic covariance function which can be written as a sum over the covering group are crucial for the efficiency of our formalism. The first one, a trivial decomposition given by (26), defines the topological signature of the covariance function. When written for the correlation matrix of the harmonic expansion coefficients, it yields the topological signature in the temperature anisotropy maps, as illustrated for the cylinder by (34). This expression shows that the topological signature is nothing but a "perturbation" of the correlation matrix corresponding to the simply connected case. Since, as discussed in Sec. IVA, these "perturbations" are small, the efficiency of the calculation follows.

The second decomposition given by (30) allows us to write the topological signature of any manifold in terms of the topological signatures of its maximal covering manifolds with cyclic covering groups. The example of the tori illustrates the power of this approach, since we can write down explicit formulas for a general torus whether its generating translations are orthogonal and/or equal. Trying to do this with the explicit use of eigenfunctions of the Laplacian (or with the method used in Appendix D) turns out to be tedious if not difficult.

Moreover, by construction, this decomposition is invariant under the symmetries of the manifold; thus it carries information on how these symmetries shall manifest in individual CMB temperature anisotropy maps, as will be discussed in Sec. VIB.

Another advantage of this second decomposition is the simplicity for writing down the power spectrum for complicated manifolds. Expressions like (47) and (50) are computationally very efficient once we have saved the power spectrum for cyclic manifolds as a function of its scale of compactification, since we have just to perform a weighted sum of power spectra for cyclic manifolds at different scales considering the multiplicity of the decomposition.

\section{B. Topological signatures}

A further advantage of splitting the correlation matrix of the multipole coefficients into its simply connected part and its topological signature is that we can identify very easily the geometric features of the signature. Although we have made explicit calculations for flat homogeneous manifolds only, qualitatively these results are general.

Universes with cylindrical topology of size $L \approx 2$ present clear alignments of their low $\ell$ modes along the direction of compactification. A dispersion map of the squares $\left\langle\left|a_{\ell m}\right|^{2}\right\rangle$, for a given low $\ell$, exhibits an axial symmetry around this direction, thus it reduces to a function of the polar angle. These dispersion maps are shown in Fig. 7.

By decomposing the covering group $\Gamma$ in cyclic subgroups, one can see that, whatever the shape of our Universe, and if it is not too large, dispersion maps (one for each individual low multipole) might show patterns of alignment. In the general case such maps are functions on the two-dimensional projective space or, by a lifting, on the 2 -sphere. Although we have shown the existence of patterns of alignment explicitly only for homogeneous flat manifolds, it follows from the exposition of the general formalism that the same conclusions hold for any manifold of constant curvature. Thus, we propose the construction of these dispersion maps in the WMAP data, and so the search for patterns of alignment, as a new method for detecting a possible nontrivial topology of our Universe.

It is interesting to comment on some features relating Levin and collaborators' proposal of pattern formation in CMB temperature maps and the results we present in this paper. The patterns proposed by Levin et al. $[12,13]$ are due to individual eigenmodes ( $k$ modes); the patterns we have identified here are due to multipole modes ( $\ell$ modes). In either case the modes compete to form their patterns in a CMB temperature map, however the observable modes in a map on the sphere are the latter, since spherical harmonics form a base on the space of functions on the sphere. On the other side, the association between real space perturbations and angular temperature fluctuations requires some averaging over the $k$ modes [6]; thus these patterns appear mixed in a map and their observation might demand more elaborated techniques.

\section{Further remarks and future research}

The formalism we have developed in this paper reveals new insights on the problem of characterizing the marks that topology leaves in $\mathrm{CMB}$ maps, and opens up new possibilities for developing further methods for unveiling the shape of our Universe. It makes explicit that the multipole alignments observed in COBE and WMAP full sky CMB temperature maps may be a manifestation of its global shape, provides details of the nature and features of these alignments, and gives at least one methodology to test this hypothesis. As a consequence, further work is much needed.

One line of further research is the implementation of our formalism in the spherical and hyperbolic cases. One way 
to do this requires first to identify the radial part of the fundamental solution (10) of the Helmholtz equation in the universal covering, as well as the analog of the "plane wave expansion" solution (17) in these geometries, and to write the expansion of the corresponding "plane waves" in spherical harmonics as in (18). The difficult part seems to be expressing the "plane wave expansion" in a suitable form to reproduce the formal steps used in the Euclidean case.

Moreover, we have to compute the topological signature of all other cyclic manifolds, in order to extend the computations to any quotient space that could be a candidate for the shape of space. We also need to include acoustic oscillations, and Doppler and finite width effects in $\Lambda \mathrm{CDM}$ models so that we could determine the relevant angular scales in cosmic topology, i.e. the angular scales at which the topological signatures appear. This is a crucial step in order to confront quantitatively the theory with real CMB maps in an efficient and rigorous way. An ultimate goal may be to implement all this methodology in a software package for public use.

The identification of the "topological signature" of the correlation matrix $\left\langle a_{\ell m} a_{\ell^{\prime} m^{\prime}}^{*}{ }^{\top}\right.$ " also opens up a path for solving a problem raised by Riazuelo et al. in [14]. The correlation matrix for a multiply connected universe is nondiagonal and, typically, $m$ dependent. In fact, this is the source of the statistical anisotropy of the CMB in these universes. However, for very large manifolds this correlation matrix becomes effectively diagonal, and equal to that corresponding to the universal covering counterpart. A natural question is raised: at what typical scales does the correlation matrix "become diagonal"? In terms of our formalism, this problem can be stated as finding the scales where the topological signature becomes observationally negligible compared to the simply connected part. A closed analysis of the topological signature might give some answers to this and related questions. For example, establishing bounds on the integral in (37) might solve the problem for the cylinder.

\section{ACKNOWLEDGMENTS}

We wish to thank Cristiane Camilo Hernandez for her invaluable help with Fig. 4, Wanderson Wanzeller for his continuous help in computational issues, and Carlos Alexandre Wuensche for showing us the papers [1] which triggered our interest in this topic. We would also like to thank the Brazilian federal institutions CBPF and INPE for warm hospitality on several occasions, and the participants of the Seminar of Cosmic Topology held monthly at IFT and the Workshop New Physics from Space held every year in Campos do Jordão, São Paulo, where we had lots of opportunities to discuss this work at several stages of its development during the past two years. W.S. HipólitoRicaldi acknowledges CAPES and G. I. Gomero acknowledges FAPESP (Contract No. 02/12328-6) for financial support.

\section{APPENDIX A: SPHERICAL HARMONICS}

In order to be self-contained and to set the notation used in the paper, in this appendix we present basic definitions, some useful formulas of spherical harmonic functions and Wigner rotation matrices, as well as some invariance properties of the correlation matrix $\left\langle a_{\ell m} a_{\ell^{\prime} m^{\prime}}^{*}\right\rangle$ under coordinate transformations. For a complete treatment of spherical harmonic functions, the reader can consult [25].

\section{Basic definitions}

Let us denote by $\mathbf{n}=(\theta, \varphi)$ a point in a 2-sphere parametrized in the usual spherical coordinates, then the spherical harmonic functions are defined as

$$
Y_{\ell m}(\mathbf{n})=\sqrt{\frac{2 \ell+1}{4 \pi} \frac{(\ell-m) !}{(\ell+m) !}} P_{\ell}^{m}(\cos \theta) e^{i m \varphi},
$$

where

$$
P_{\ell}^{m}(x)=(-1)^{m}\left(1-x^{2}\right)^{m / 2} \frac{d^{m}}{d x^{m}} P_{\ell}(x)
$$

are the associated Legendre functions with non-negative index $0 \leq m \leq \ell$, and with

$$
P_{\ell}(x)=\frac{1}{2^{\ell} \ell !} \frac{d^{\ell}}{d x^{\ell}}\left(x^{2}-1\right)^{\ell}
$$

being the Legendre polynomials. The associated Legendre functions with negative index $m$ are defined by

$$
P_{\ell}^{-m}(x)=(-1)^{m} \frac{(\ell-m) !}{(\ell+m) !} P_{\ell}^{m}(x) .
$$

Moreover, it is often convenient to introduce the normalized associated Legendre functions

$$
\mathcal{P}_{\ell}^{m}(x)=\sqrt{\frac{2 \ell+1}{2} \frac{(\ell-m) !}{(\ell+m) !}} P_{\ell}^{m}(x) .
$$

It can easily be seen that the Legendre polynomial $P_{\ell}(x)$ is an $\ell$ th degree polynomial of parity $\ell$, and thus the associated Legendre function $P_{\ell}^{m}(x)$ is a function of parity $\ell-m$. It follows that the function $\mathcal{P}_{\ell \ell^{\prime} m}(x)$ defined in (41) is an $\left(\ell+\ell^{\prime}\right)$ th degree polynomial of parity $\ell+\ell^{\prime}$, and thus the expression for $F_{\ell \ell^{\prime}}^{m}(x)$ in (38), which is evaluated in Appendix C, contains only even polynomials.

Spherical harmonics form a complete orthonormal set of functions on the sphere, thus their most common application is in the expansion of functions, like a CMB temperature anisotropy map, in multipoles as in (11), where the coefficients $a_{\ell m}$, called the multipole coefficients, are given by

$$
a_{\ell m}=\int_{\mathbb{S}^{2}} d \Omega \frac{\delta T}{T}(\mathbf{n}) Y_{\ell m}^{*}(\mathbf{n}) .
$$

Since the temperature map is a real function on the sphere, 
the multipole coefficients obey the constraint $a_{\ell m}^{*}=$ $(-1)^{m} a_{\ell,-m}$.

A very useful formula is given by the addition theorem for spherical harmonics:

$$
P_{\ell}\left(\mathbf{n} \cdot \mathbf{n}^{\prime}\right)=\frac{4 \pi}{2 \ell+1} \sum_{m=-\ell}^{\ell} Y_{\ell m}(\mathbf{n}) Y_{\ell m}^{*}\left(\mathbf{n}^{\prime}\right),
$$

which for the particular case $\mathbf{n}=\mathbf{n}^{\prime}$ yields the identity

$$
\sum_{m=-\ell}^{\ell}\left[\mathcal{P}_{\ell}^{m}(x)\right]^{2}=\frac{2 \ell+1}{2}
$$

\section{Wigner $D$ functions}

On several occasions it is convenient to rotate the sphere and compute the multipole coefficients in this new coordinate system. This can be achieved by means of the Wigner $D$ functions which can be defined operationally as the functions $D_{m m_{1}}^{\ell}(R)$ such that, for any rotation $R \in$ $\mathrm{SO}(3)$, then

$$
Y_{\ell m}(R \mathbf{n})=\sum_{m_{1}} D_{m m_{1}}^{\ell}(R) Y_{\ell m_{1}}(\mathbf{n}) .
$$

In this case, it can be shown that the multipole coefficients of the temperature anisotropy map in the rotated reference frame are

$$
\tilde{a}_{\ell m}=\sum_{m_{1}} D_{m m_{1}}^{* \ell}(R) a_{\ell m_{1}} .
$$

This expression can be used to compute the correlation matrix of the $a_{\ell m}$ 's in a rotated frame simply as

$$
\left\langle a_{\ell m} a_{\ell^{\prime} m^{\prime}}^{*}\right\rangle_{R}=\sum_{m_{1}, m_{1}^{\prime}} D_{m m_{1}}^{* \ell}(R) D_{m^{\prime} m_{1}^{\prime}}^{\ell^{\prime}}(R)\left\langle a_{\ell m_{1}} a_{\ell^{\prime} m_{1}^{\prime}}^{*}\right\rangle .
$$

The Wigner $D$ functions take a very simple form when we express the rotation matrix $R$ in terms of its Euler angles as

$$
R(\alpha, \beta, \gamma)=R_{z}(\alpha) \cdot R_{y}(\beta) \cdot R_{z}(\gamma) .
$$

Indeed, for this decomposition we have

$$
D_{m m^{\prime}}^{\ell}(R(\alpha, \beta, \gamma))=e^{i\left(m \alpha+m^{\prime} \gamma\right)} d_{m m^{\prime}}^{\ell}(\beta),
$$

where $d_{m m^{\prime}}^{\ell}(\beta)=D_{m m^{\prime}}^{\ell}\left(R_{y}(\beta)\right)$ is a real matrix with the following symmetries:

$$
\begin{aligned}
d_{m m^{\prime}}^{\ell}(\beta) & =(-1)^{m-m^{\prime}} d_{m^{\prime} m}^{\ell}(\beta), \\
d_{m m^{\prime}}^{\ell}(\beta) & =d_{-m^{\prime},-m}^{\ell}(\beta), \\
d_{m m^{\prime}}^{\ell}(\pi-\beta) & =(-1)^{\ell-m^{\prime}} d_{-m, m^{\prime}}^{\ell}(\beta), \\
d_{m m^{\prime}}^{\ell}(-\beta) & =(-1)^{m^{\prime}-m} d_{m, m^{\prime}}^{\ell}(\beta) .
\end{aligned}
$$

There exist several explicit and recursive formulas to compute these matrices (see [25]). A very efficient recursive procedure can be found in [26]. The following formula will be enough to reproduce the results presented in this paper.

$$
\begin{aligned}
d_{m m^{\prime}}^{\ell}(\beta)= & \sqrt{(\ell+m) !(\ell-m) !\left(\ell+m^{\prime}\right) !\left(\ell-m^{\prime}\right) !} \sum_{k}(-1)^{k} \\
& \times \frac{\left(\cos \frac{\beta}{2}\right)^{2 \ell-2 k+m-m^{\prime}}\left(\sin \frac{\beta}{2}\right)^{2 k-m+m^{\prime}}}{k !(\ell+m-k) !\left(\ell-m^{\prime}-k\right) !\left(m^{\prime}-m+k\right) !},
\end{aligned}
$$

where the sum in $k$ is evaluated whenever the arguments inside the factorials are non-negative.

\section{Symmetry considerations}

Some consequences of the symmetries of the quotient manifold on the invariance structure of the correlation matrix of the $a_{\ell m}$ 's can be deduced directly from the transformation rules of the spherical harmonic functions under coordinate transformations. The results obtained in this way are formal, generic, and are very useful in practical computations. We end this appendix by deducing the invariance properties the correlation matrix must have, given some symmetries of the corresponding quotient manifold. These invariance properties have been used in [14] to simplify the correlation matrix for the 3-torus, however we want to remark here that they are general and do not depend on the geometry of the universal covering space.

Let us begin with the invariance properties of $\left\langle a_{\ell m} a_{\ell^{\prime} m^{\prime}}^{*}\right\rangle^{\Gamma}$ under rotations around the $z$ axis. Under a rotation $R_{z}(\alpha): \varphi \rightarrow \varphi+\alpha$, the function $Y_{\ell m}(\mathbf{n})$ transforms as

$$
Y_{\ell m}\left(R_{z}(\alpha) \mathbf{n}\right)=e^{i m \alpha} Y_{\ell m}(\mathbf{n}) .
$$

As a consequence, the transformation rules for the multipole coefficients of a CMB temperature map are of the form $\tilde{a}_{\ell m}=e^{-i m \alpha} a_{\ell m}$, and so the correlation matrix transforms under this rotation as

$$
\left\langle a_{\ell m} a_{\ell^{\prime} m^{\prime}}^{*}\right\rangle_{R_{z}(\alpha)}^{\Gamma}=e^{i\left(m^{\prime}-m\right) \alpha}\left\langle a_{\ell m} a_{\ell^{\prime} m^{\prime}}^{*} \Gamma^{\Gamma} .\right.
$$

We extract two consequences out of (A5). First, if the quotient space is invariant under a rotation of $\alpha=2 \pi / s$ around the $z$ axis, then the correlation matrix must be zero unless $m=m^{\prime} \bmod s$. Second, if the quotient space is invariant under "any" rotation around the $z$ axis, the correlation matrix must be zero unless $m=m^{\prime}$. In practice, if we take our coordinate system such that the fundamental polyhedron of the quotient manifold is oriented so that it is invariant under a $2 \pi / s$ rotation around the polar axis, the correlation matrix will present a factor $\delta_{m m^{\prime}}^{\bmod (s)}$, and correspondingly, if the orientation is such that the polyhedron is invariant under arbitrary rotations around the $z$ axis, the correlation matrix will present a factor $\delta_{m m^{\prime}}$.

Let us now take a look at invariance under the inversion transformation $P: \mathbf{n} \rightarrow-\mathbf{n}$. Under this transformation the spherical harmonic functions change as

$$
Y_{\ell m}(P \mathbf{n})=(-1)^{\ell} Y_{\ell m}(\mathbf{n})
$$


thus the multipole coefficients $a_{\ell m}$ change as $\tilde{a}_{\ell m}=$ $(-1)^{\ell} a_{\ell m}$, and as a consequence the transformation rule for the correlation matrix is

$$
\left\langle a_{\ell m} a_{\ell^{\prime} m^{\prime}}^{*}\right\rangle_{P}^{\Gamma}=(-1)^{\ell+\ell^{\prime}}\left\langle a_{\ell m} a_{\ell^{\prime} m^{\prime}}^{*}\right\rangle^{\Gamma} .
$$

Thus, if the fundamental polyhedron is oriented such that it appears invariant under the parity transformation, the correlation matrix must be zero unless $\ell=\ell^{\prime} \bmod 2$, i.e., the correlation matrix will present a factor $\delta_{\ell \ell^{\prime}}^{\bmod (2)}$.

To end this section, let us consider the reflection on the $y=0$ plane. This operation changes only the azimuthal angle as $P_{y}: \varphi \rightarrow-\varphi$, thus the transformation rule for the spherical harmonics is

$$
Y_{\ell m}\left(P_{y} \mathbf{n}\right)=Y_{\ell m}^{*}(\mathbf{n}),
$$

the multipole coefficients $a_{\ell m}$ change as $\tilde{a}_{\ell m}=a_{\ell m}^{*}$, and thus, the transformation rule for the correlation matrix is

$$
\left\langle a_{\ell m} a_{\ell^{\prime} m^{\prime}}^{*}\right\rangle_{P_{y}}^{\Gamma}=\left\langle a_{\ell m} a_{\ell^{\prime} m^{\prime}}^{*}\right\rangle^{* \Gamma} .
$$

It immediately follows that if the fundamental polyhedron is oriented such that it appears invariant under the reflection on the $y=0$ plane, the correlation matrix must be real.

\section{APPENDIX B: CLAUSEN FUNCTIONS}

In this appendix we briefly present some computational aspects of the theory of Clausen functions, as far as we need them for our purposes. Clausen functions are periodic functions of period $2 \pi$. There are two kinds of Clausen functions, the $\varphi$ class and the $\psi$ class. Clausen $\varphi$ functions can be expressed in terms of polynomials, while Clausen $\psi$ functions involve higher transcendental functions, the socalled Clausen integrals. Fortunately, we are interested exclusively in the Clausen $\varphi$ functions, thus we will develop the details of the theory only for them. The Clausen $\varphi$ functions are defined as

$$
\varphi_{2 s-1}(x)=\sum_{n=1}^{\infty} \frac{\sin n x}{n^{2 s-1}}, \quad \varphi_{2 s}(x)=\sum_{n=1}^{\infty} \frac{\cos n x}{n^{2 s}},
$$

for $s=1,2, \ldots$, and can be calculated recursively with the formulas,

$$
\begin{aligned}
\varphi_{2 s}(x) & =\zeta(2 s)-\int_{0}^{x} \varphi_{2 s-1}(y) d y, \\
\varphi_{2 s+1}(x) & =\int_{0}^{x} \varphi_{2 s}(y) d y,
\end{aligned}
$$

where

$$
\zeta(s)=\sum_{n=1}^{\infty} \frac{1}{n^{s}}
$$

is the Riemann zeta function.

These recurrence relations are complemented by the initial condition

$$
\begin{aligned}
\varphi_{1}(x)= & \sum_{n=1}^{\infty} \frac{\sin n x}{n} \\
= & \frac{1}{2} \sum_{q \in \mathbb{Z}}[(2 q+1) \pi-x] \\
& \times \Theta(x-2 \pi q) \Theta(2 \pi(q+1)-x),
\end{aligned}
$$

where $\Theta(x)$ is the Heaviside step function. Formula (B3) can be verified by computing the Fourier series of the second right-hand side.

Since the Clausen functions are periodic of period $2 \pi$, we can write

$$
\varphi_{s}(x)=\sum_{q \in \mathbb{Z}} f_{s}(x-2 \pi q) \Theta(x-2 \pi q) \Theta(2 \pi(q+1)-x),
$$

with $f_{1}(x)=\frac{1}{2}(\pi-x)$. The recurrence formulas (B2) yield the following expressions for the Clausen functions in the period $[0,2 \pi]$ :

$$
\begin{aligned}
f_{2 s+1}(x)= & \sum_{r=0}^{s-1} \frac{(-1)^{r}}{(2 r+1) !} \zeta(2(s-r)) x^{2 r+1} \\
& +\frac{(-1)^{s}}{2}\left(\frac{\pi x^{2 s}}{(2 s) !}-\frac{x^{2 s+1}}{(2 s+1) !}\right)
\end{aligned}
$$

for $s=0,1,2, \ldots$, and

$$
\begin{aligned}
f_{2 s}(x)= & \sum_{r=0}^{s-1} \frac{(-1)^{r}}{(2 r) !} \zeta(2(s-r)) x^{2 r} \\
& +\frac{(-1)^{s}}{2}\left(\frac{\pi x^{2 s-1}}{(2 s-1) !}-\frac{x^{2 s}}{(2 s) !}\right)
\end{aligned}
$$

for $s=1,2,3, \ldots$.

From the definitions (B1) we get $f_{2 s+1}(\pi)=0$, which can be used to obtain a recurrence formula for the Riemann zeta function of even argument,

$$
\zeta(2 s)=\sum_{r=1}^{s-1} \frac{(-1)^{r+1}}{(2 r+1) !} \zeta(2(s-r)) \pi^{2 r}-\frac{(-1)^{s} s}{(2 s+1) !} \pi^{2 s} .
$$

Writing $\zeta(2 s)=g_{2 s}(0) \pi^{2 s}$, and substituting this into (B6), we have

$$
g_{2 s}(0)=\sum_{r=1}^{s-1} \frac{(-1)^{r+1}}{(2 r+1) !} g_{2(s-r)}(0)-\frac{(-1)^{s} s}{(2 s+1) !} .
$$

The convenience for introducing this notation will be apparent in what follows.

We will now seek for generalizations of the formulas (B4) and (B5), i.e., we look for explicit expressions for the Clausen functions in the $q$ th interval $[2 \pi q, 2 \pi(q+1)]$. Since the Clausen functions satisfy the periodicity conditions $\varphi_{2 s-1}(2 \pi q)=0$ and $\varphi_{2 s}(2 \pi q)=\zeta(2 s)$, the recur- 
rence relations $(\mathrm{B} 2)$ can be rewritten in the form

$$
\begin{aligned}
\varphi_{2 s}(x) & =\zeta(2 s)-\int_{2 \pi q}^{x} \varphi_{2 s-1}(y) d y, \\
\varphi_{2 s+1}(x) & =\int_{2 \pi q}^{x} \varphi_{2 s}(y) d y,
\end{aligned}
$$

Defining the polynomials $f_{s}^{q}(x)=f_{s}(x-2 \pi q)$, we notice that $\varphi_{s}(x)$ coincides with $f_{s}^{q}(x)$ in the interval $[2 \pi q, 2 \pi(q+1)]$. This fact, and the expressions (B7), allow us to write recurrence formulas analog to (B2) for the polynomials $f_{s}^{q}(x)$ as follows:

$$
\begin{gathered}
f_{2 s}^{q}(x)=g_{2 s}(q) \pi^{2 s}-\int_{0}^{x} f_{2 s-1}^{q}(y) d y, \\
f_{2 s+1}^{q}(x)=g_{2 s+1}(q) \pi^{2 s+1}+\int_{0}^{x} f_{2 s}^{q}(y) d y,
\end{gathered}
$$

where

$$
\begin{aligned}
g_{2 s}(q) & =g_{2 s}(0)+\frac{1}{\pi^{2 s}} \int_{0}^{2 \pi q} f_{2 s-1}^{q}(y) d y, \\
g_{2 s+1}(q) & =-\frac{1}{\pi^{2 s+1}} \int_{0}^{2 \pi q} f_{2 s}^{q}(y) d y,
\end{aligned}
$$

with initial conditions, given by the first Clausen function, $f_{1}^{q}(x)=g_{1}(q) \pi-\frac{x}{2}$ and $g_{1}(q)=q+\frac{1}{2}$.

The expressions (B8) can be written in a unified way as

$$
f_{s}^{q}(x)=g_{s}(q) \pi^{s}-(-1)^{s} \int_{0}^{x} f_{s-1}^{q}(y) d y .
$$

Using this expression, we readily obtain the explicit formula, which is the generalization of (B4) and (B5) we were looking for:

$$
f_{s}^{q}(x)=\sum_{r=0}^{s-1} \frac{(-1)^{\mu(r, s)}}{r !} g_{s-r}(q) \pi^{s-r} x^{r}-\frac{(-1)^{\mu(s, 1)}}{2} \frac{x^{s}}{s !},
$$

where

$$
\mu(r, s)=\left\lfloor\frac{r}{2}+\frac{1+(-1)^{s}}{4}\right\rfloor,
$$

and $\lfloor x\rfloor$ is the floor function of $x$, i.e., the largest integer smaller than $x$.

The expressions (B9) can also be written in a unified way as

$$
g_{s}(q)=g_{s}(0)+\frac{(-1)^{s}}{\pi^{s}} \int_{0}^{2 \pi q} f_{s-1}^{q}(y) d y,
$$

where

$$
g_{s}(0)= \begin{cases}\frac{\zeta(s)}{\pi^{s}} & \text { if } s \text { is even } \\ 0 & \text { if } s>1 \text { is odd }\end{cases}
$$

From this we get the expression analogous to (B10):

$$
\begin{aligned}
g_{s}(q)= & g_{s}(0)+(-1)^{s}\left[\sum_{r=1}^{s-1}(-1)^{\mu(r-1, s-1)} \frac{2^{r}}{r !} g_{s-r}(q) q^{r}\right. \\
& \left.-(-1)^{\mu(s-1,1)} \frac{2^{s-1}}{s !} q^{s}\right] .
\end{aligned}
$$

The polynomials $g_{s}(q)$ can also be written in the canonical form

$$
g_{s}(q)=\sum_{k=0}^{s} A_{k}^{s} q^{k},
$$

where the coefficients are given by $A_{0}^{s}=g_{s}(0)$,

$$
A_{n}^{s}=(-1)^{s} \sum_{r=1}^{n}(-1)^{\mu(r-1, s-1)} \frac{2^{r}}{r !} A_{n-r}^{s-r}
$$

for $0<n<s$, and

$A_{s}^{s}=(-1)^{s}\left[\sum_{r=1}^{s-1}(-1)^{\mu(r-1, s-1)} \frac{2^{r}}{r !} A_{s-r}^{s-r}-(-1)^{\mu(s-1,1)} \frac{2^{s-1}}{s !}\right]$,

with initial conditions $A_{0}^{1}=\frac{1}{2}$ and $A_{1}^{1}=1$. These coefficients are obtained by just introducing (B12) into (B11) and collecting terms.

\section{APPENDIX C: THE FUNCTION $F_{\ell \ell^{\prime}}^{m}(x)$}

In this appendix we evaluate the function $F_{\ell \ell^{\prime}}^{m}(x)$ given by (38). We first observe (see Appendix A) that the function $\mathcal{P}_{\ell \ell^{\prime} m}(x)$, given by $(41)$, is an even polynomial of $(\ell+$ $\left.\ell^{\prime}\right)$ degree. Thus, we begin by considering the integral

$$
I(\alpha)=\int_{-1}^{1} P(y) \cos \alpha y d y,
$$

where $P(y)$ is an even analytical function. Integrating successively by parts, we get

$$
\begin{aligned}
I(\alpha)= & 2\left[\frac{\sin \alpha}{\alpha} \sum_{s=0}^{\infty} \frac{(-1)^{s}}{\alpha^{2 s}} P^{(2 s)}(1)\right. \\
& \left.+\frac{\cos \alpha}{\alpha^{2}} \sum_{s=0}^{\infty} \frac{(-1)^{s}}{\alpha^{2 s}} P^{(2 s+1)}(1)\right],
\end{aligned}
$$

where $P^{(k)}(x)$ is the $k$ th derivative of $P(x)$.

Making $\alpha=n x$ and $P(x)=\mathcal{P}_{\ell \ell^{\prime} m}(x)$ in $(\mathrm{C} 1)$, substituting (C1) in (38), and performing the sum in $n$, we get

$$
\begin{aligned}
F_{\ell \ell^{\prime}}^{m}(x)= & 4 \sum_{s=0}^{\left(\ell+\ell^{\prime}\right) / 2}(-1)^{s}\left[\frac{\mathcal{P}_{\ell \ell^{\prime} m}^{(2 s)}(1)}{x^{2 s+1}} \varphi_{2 s+1}(x)\right. \\
& \left.+\frac{\mathcal{P}_{\ell \ell^{\prime} m}^{(2 s+1)}(1)}{x^{2 s+2}} \varphi_{2 s+2}(x)\right],
\end{aligned}
$$

where $\varphi_{k}(x)$ is the $k$ th Clausen $\varphi$ function defined in Appendix B. 
Since the Clausen functions are periodic functions of period $2 \pi$, analytic in each period, it follows that $F_{\ell \ell^{\prime}}^{m}(x)$ is a piecewise continuous function, analytic in each period as well. Thus we will now show how the explicit expression for $F_{\ell \ell^{\prime}}^{m}(x)$, in the $q$ th interval $[2 \pi q, 2 \pi(q+1)]$, given in (40), comes out.

Introducing the explicit form for the Clausen $\varphi$ functions (B10), in the sum of (C2) yields a huge expression, but a close inspection reveals that it is a polynomial in $\pi / x$. The independent term is simply

$$
\begin{aligned}
-\frac{1}{2} \sum_{s=0}^{\left(\ell+\ell^{\prime}\right) / 2} \frac{(-1)^{s}}{(s+1) !} \mathcal{P}_{\ell \ell^{\prime} m}^{(s)}(1) & =-\frac{1}{4} \int_{-1}^{1} \mathcal{P}_{\ell \ell^{\prime} m}(x) d x \\
& =-\frac{1}{4} \delta_{\ell \ell^{\prime}},
\end{aligned}
$$

where the first equality can be deduced by writing the Taylor expansion of the integrand of the right-hand side, and integrating. On the other hand, summing up all the coefficients of the $(r+1)$ th odd term, and proceeding as before, we have this term equal to

$$
(-1)^{r} \mathcal{P}_{\ell \ell^{\prime} m}^{(2 r)}(0) g_{2 r+1}(q)\left(\frac{\pi}{x}\right)^{2 r+1},
$$

while the $(r+1)$ th even term is equal to

$$
(-1)^{r} \mathcal{P}_{\ell \ell^{\prime} m}^{(2 r+1)}(0) g_{2 r+2}(q)\left(\frac{\pi}{x}\right)^{2 r+2},
$$

which by the parity of $\mathcal{P}_{\ell \ell^{\prime} m}(x)$ is zero. Summing up all the terms we finally get (39) and (40).

\section{APPENDIX D: KNOWN RESULTS FOR CLOSED FLAT 3-MANIFOLDS}

In this section we briefly show how we can obtain the formulas for the correlation matrix of the $a_{\ell m}$ 's and the angular power spectrum, currently available in the literature, for some closed flat manifolds, as well as a simple generalization, i.e., considering the observer out of the axis of rotations of the screw motions of the covering group. We present explicit derivations and formulas for the correlation matrix $\left\langle a_{\ell m} a_{\ell^{\prime} m^{\prime}}^{*}\right\rangle$, as well as for the power spectrum, in order to allow the interested reader to perform their own simulations confidently.

We first give a brief description of flat orientable closed 3 -manifolds and their covering groups. The versions of the diffeomorphic and isometric classifications of flat 3manifolds we present here were given by Wolf in [27], and previous descriptions in the context of cosmic topology were given in [28] (see $[4,16]$ for alternative descriptions).

There are six diffeomorphic classes of compact orientable Euclidean 3-manifolds. The generators for the covering groups of the first five classes, $G_{1}-G_{5}$, are $\gamma_{1}=(I, \mathbf{a}), \gamma_{2}=(I, \mathbf{b})$, and $\gamma_{3}=\left(A_{i}, \mathbf{c}\right)$, where $A_{1}=I$ is the identity and

$$
\begin{array}{rlrl}
A_{2} & =\left(\begin{array}{ccc}
-1 & 0 & 0 \\
0 & -1 & 0 \\
0 & 0 & 1
\end{array}\right), & A_{4}=\left(\begin{array}{ccc}
0 & -1 & 0 \\
1 & 0 & 0 \\
0 & 0 & 1
\end{array}\right), \\
A_{3}=\left(\begin{array}{ccc}
0 & -1 & 0 \\
1 & -1 & 0 \\
0 & 0 & 1
\end{array}\right), & A_{5}=\left(\begin{array}{ccc}
0 & -1 & 0 \\
1 & 1 & 0 \\
0 & 0 & 1
\end{array}\right),
\end{array}
$$

for the classes $G_{1}-G_{5}$ respectively. It is important to remark that these matrices for the rotations are written in the basis formed by the set $\{\mathbf{a}, \mathbf{b}, \mathbf{c}\}$ of linearly independent vectors. Thus, the torus $G_{1}$ is generated by three independent translations, while for the other manifolds the generators are two independent translations and a screw motion along a linearly independent direction. The manifold $G_{6}$ is the most involved since their generators are all screw motions. In the following, we present some general considerations concerning the classes $G_{1}-G_{5}$ only.

For space forms of the classes $G_{2}-G_{5}$, the following facts are easily derivable (see [28] for details):

(1) The vector $\mathbf{c}$ is orthogonal to both $\mathbf{a}$ and $\mathbf{b}$.

(2) The angle between $\mathbf{a}$ and $\mathbf{b}$ is a free parameter for the class $G_{2}$, while its value is fixed to be $2 \pi / 3$, $\pi / 2$, and $\pi / 3$ for the classes $G_{3}, G_{4}$, and $G_{5}$, respectively.

(3) Denoting by $|\mathbf{c}|$ the length of the vector $\mathbf{c}$, and similarly for any other vector, one has that $|\mathbf{a}|=$ $|\mathbf{b}|$ for the classes $G_{3}-G_{5}$, while both lengths are independent free parameters in the class $G_{2}$. Moreover, in all classes $G_{2}-G_{5},|\mathbf{c}|$ is an independent free parameter.

(4) Denoting the canonical unitary basis vectors in Euclidean space by $\left\{\hat{\mathbf{e}}_{x}, \hat{\mathbf{e}}_{y}, \hat{\mathbf{e}}_{z}\right\}$, one can always write $\mathbf{a}=|\mathbf{a}| \hat{\mathbf{e}}_{x}, \mathbf{b}=|\mathbf{b}| \cos \varphi \hat{\mathbf{e}}_{x}+|\mathbf{b}| \sin \varphi \hat{\mathbf{e}}_{y}$, and $\mathbf{c}=$ $|\mathbf{c}| \hat{\mathbf{e}}_{z}$, for the basis $\{\mathbf{a}, \mathbf{b}, \mathbf{c}\}$, where $\varphi$ is the angle between $\mathbf{a}$ and $\mathbf{b}$, as established in the item 2 .

Thus in dealing with manifolds of classes $G_{2}-G_{5}$, the axis of rotation of the generator screw motion can be taken to be the $z$ axis, and the orthogonal part of this generator, in the basis $\left\{\hat{\mathbf{e}}_{x}, \hat{\mathbf{e}}_{y}, \hat{\mathbf{e}}_{z}\right\}$, is

$$
A=\left(\begin{array}{ccc}
\cos \alpha & -\sin \alpha & 0 \\
\sin \alpha & \cos \alpha & 0 \\
0 & 0 & 1
\end{array}\right)
$$

with $\alpha=\pi, 2 \pi / 3, \pi / 2$, and $\pi / 3$, respectively. Since the axis of rotation passes through the origin, the translational part of the generator $\gamma_{3}$ is $\mathbf{c}=\left(0,0, L_{z}\right)$, where we have put $|\mathbf{c}|=L_{z}$ as is usual in cosmic topology.

However, in cosmological applications we need to consider the arbitrariness of the position of the observer inside space. Thus if the axis of rotation is at a distance $\rho$ from the origin (the observer), and its intersection with the horizontal plane makes an angle $\phi$ with the positive $x$ axis, the 
translational part of the screw motion $\gamma_{3}=(A, \mathbf{c})$ is

$$
\begin{aligned}
\mathbf{c}= & \rho[\cos \phi-\cos (\phi+\alpha)] \hat{\mathbf{e}}_{x} \\
& +\rho[\sin \phi-\sin (\phi+\alpha)] \hat{\mathbf{e}}_{y}+L_{z} \hat{\mathbf{e}}_{z} .
\end{aligned}
$$

In order to perform calculations of the topological signature of $\mathrm{CMB}$ temperature maps, we need to write the covering group for the manifold under study in a compact form. For a torus $G_{1}$ the problem is trivial, since the covering group is generated by three independent translations, and thus any two isometries commute (see Sec. D 1 below), while the covering groups for the other closed flat manifolds are noncommutative since they contain screw motions.

The generators of the covering groups for the classes $G_{2}-G_{5}$ satisfy certain relations of the form

$$
\gamma_{3} \gamma_{1}^{n_{1}} \gamma_{2}^{n_{2}}=\gamma_{1}^{m_{1}} \gamma_{2}^{m_{2}} \gamma_{3}
$$

where $n_{1}, n_{2}, m_{1}, m_{2} \in \mathbb{Z}$, and they hold whether the axis of rotation passes through the origin or not. It follows that a generic isometry can always be put in the form

$$
\gamma=\gamma_{1}^{n_{1}} \gamma_{2}^{n_{2}} \gamma_{3}^{n_{3}}
$$

with $\gamma_{1}^{n_{1}}=\left(I, n_{1} \mathbf{a}\right), \gamma_{2}^{n_{2}}=\left(I, n_{2} \mathbf{b}\right)$, and

$$
\gamma_{3}^{n_{3}}=\left(A^{h}, n_{3} \mathbf{c}_{\|}+\mathcal{O}_{h} \mathbf{c}_{\perp}\right),
$$

where $A$ is given by (D1), $\alpha=2 \pi / s, n_{3}=s q+h$, with $q$ and $h$ integers such that $0<h \leq s$, the parameter $s$ being $2,3,4$, and 6 corresponding to $G_{2}, G_{3}, G_{4}$, and $G_{5}$, respectively,

$$
\mathcal{O}_{h}=\sum_{j=0}^{h-1} A^{j}
$$

$\mathbf{c}_{\|}=L_{z} \hat{\mathbf{e}}_{z}, \quad$ and $\quad \mathbf{c}_{\perp}=\rho[\cos \phi-\cos (\phi+\alpha)] \hat{\mathbf{e}}_{x}+$ $\rho[\sin \phi-\sin (\phi+\alpha)] \hat{\mathbf{e}}_{y}$.

It is now straightforward to compute both the correlation matrix $\left\langle a_{\ell m} a_{\ell^{\prime} m^{\prime}}^{*}\right\rangle$ and the angular power spectrum $C_{\ell}$. They all have a simple structure. We first describe the general procedure for obtaining these expressions and present the results in a unified form. We finally specify each case separately. Note that, due to (D2), in all of our calculations we are considering that the observer may be off an axis of rotation of the screw motions of $\Gamma$.

Upon introducing (D3) into (25), we transform the series of exponentials in a series of Dirac's delta functions by using (32). The integration of (23) is then immediate in Cartesian coordinates, the general result being

$$
\begin{aligned}
\left\langle a_{\ell m} a_{\ell^{\prime} m^{\prime}}^{*}\right\rangle= & \frac{(4 \pi)^{2}}{V} i^{\ell-\ell^{\prime}} \sum_{\mathbf{p} \in \overrightarrow{\mathbb{Z}}^{3}} \frac{1}{\beta^{3}} \Psi_{\ell \ell^{\prime}}(2 \pi \beta) Y_{\ell^{\prime} m^{\prime}}\left(\mathbf{n}_{\vec{\beta}}\right) \\
& \times Y_{\ell m}^{*}\left(\mathbf{n}_{\vec{\beta}}\right) f_{m^{\prime}}^{\Gamma}(2 \pi \vec{\beta}),
\end{aligned}
$$

where $V$ is the volume of the manifold, and $\hat{\mathbb{Z}}^{3}=\mathbb{Z}^{3} \backslash$
$(0,0,0)$, since the term corresponding to $\mathbf{p}=0$ represents a constant perturbation, and thus is neglected.

The function

$$
f_{m}^{\Gamma}(\mathbf{k})=\frac{1}{s}\left[1+\sum_{h=1}^{s-1} \omega_{s}^{-h m} e^{-i \mathbf{k} \cdot \mathcal{O}_{h} \mathbf{c}}\right],
$$

where $\omega_{s}$ is the first complex sth root of unity, is a complex modulating term characteristic of the geometry and topology of the spatial section of the universe model, and depends only on the screw motion generators. The vector $\vec{\beta}(\mathbf{p})$ comes from the discretization of the wave vector $\mathbf{k}$ due to the Dirac's deltas (each $2 \pi \beta$ is an eigenvalue of the Laplacian operator), and $\mathbf{n}_{\vec{\beta}}$ is the unit vector in the direction of $\vec{\beta}$.

Using the property $\left\langle a_{\ell m} a_{\ell^{\prime} m^{\prime}}^{*}\right\rangle=\left\langle a_{\ell^{\prime} m^{\prime}} a_{\ell m}^{*}\right\rangle^{*}$, one can easily show, by resumming the series, that the variances of the multipole moments can be put in the general form

$$
\left\langle\left|a_{\ell m}\right|^{2}\right\rangle=\frac{(4 \pi)^{2}}{V} \sum_{\mathbf{p} \in \hat{\mathbb{Z}}^{3}} \frac{1}{\beta^{3}} \Psi_{\ell \ell}(2 \pi \beta)\left|Y_{\ell m}\left(\mathbf{n}_{\vec{\beta}}\right)\right|^{2} \Re\left(f_{m}^{\Gamma}(2 \vec{\pi} \beta)\right),
$$

where $\Re$ stands for the real part of a complex number. The angular power spectrum is then

$$
C_{\ell}=\frac{4 \pi}{V} \sum_{\mathbf{p} \in \hat{\mathbb{Z}}^{3}} \frac{1}{\beta^{3}} \Psi_{\ell \ell}(2 \pi \beta) \Xi_{\ell}(2 \pi \vec{\beta}),
$$

where

$$
\Xi_{\ell}(\mathbf{k})=\frac{4 \pi}{2 \ell+1} \sum_{m=-l}^{\ell}\left|Y_{\ell m}\left(\mathbf{n}_{\mathbf{k}}\right)\right|^{2} \Re\left(f_{m}^{\Gamma}(\mathbf{k})\right)
$$

can be evaluated using the addition theorem for spherical harmonics yielding

$$
\Xi_{\ell}(\mathbf{k})=\frac{1}{s}\left[1+\sum_{h=1}^{s-1} P_{\ell}\left(\cos \theta_{\mathbf{k}, h}\right) \cos \left(\mathbf{k} \cdot \mathcal{O}_{h} \mathbf{c}\right)\right],
$$

where

$$
\cos \theta_{\mathbf{k}, h}=\cos ^{2} \theta_{\mathbf{k}}+\sin ^{2} \theta_{\mathbf{k}} \cos \frac{2 \pi h}{s} .
$$

\section{Rectangular torus $G_{1}$}

The generators for the rectangular torus are the translations $\mathbf{a}=L_{x} \hat{\mathbf{e}}_{x}, \mathbf{b}=L_{y} \hat{\mathbf{e}}_{y}$, and $\mathbf{c}=L_{z} \hat{\mathbf{e}}_{z}$, thus a generic isometry of its covering group can be written as $\gamma=(I, \mathbf{r})$, with $\mathbf{r}=n_{x} L_{x} \hat{\mathbf{e}}_{x}+n_{y} L_{y} \hat{\mathbf{e}}_{y}+n_{z} L_{z} \hat{\mathbf{e}}_{z}$, and $n_{x}, n_{y}, n_{z} \in \mathbb{Z}$, i.e., the covering group of $G_{1}$ is parametrized by $\mathbb{Z}^{3}$.

It follows immediately that, for a rectangular torus, the expression (25) takes the form 


$$
\begin{aligned}
Y_{\ell m}^{\Gamma}(\mathbf{k})= & \sum_{\mathbf{n} \in \mathbb{Z}^{3}} e^{-i\left(n_{x} k_{x} L_{x}+n_{y} k_{y} L_{y}+n_{z} k_{z} L_{z}\right)} Y_{\ell m}\left(\mathbf{n}_{\mathbf{k}}\right) \\
= & (2 \pi)^{3} \sum_{\mathbf{p} \in \mathbb{Z}^{3}} \delta\left(k_{x} L_{x}-2 \pi p_{x}\right) \delta\left(k_{y} L_{y}\right. \\
& \left.-2 \pi p_{y}\right) \delta\left(k_{z} L_{z}-2 \pi p_{z}\right) Y_{\ell m}\left(\mathbf{n}_{\mathbf{k}}\right) .
\end{aligned}
$$

Following the procedure described above one gets $f_{m}^{\Gamma}(\mathbf{k})=1$, and $\beta_{x}=p_{x} / L_{x}, \beta_{y}=p_{y} / L_{y}$, and $\beta_{z}=$ $p_{z} / L_{z}$. In particular, we have the well-known result

$$
C_{\ell}=\frac{4 \pi}{V} \sum_{\mathbf{p} \in \hat{\mathbb{Z}}^{3}} \frac{1}{\beta^{3}} \Psi_{\ell \ell}(2 \pi \beta) .
$$

\section{Rectangular $\boldsymbol{G}_{2}$}

The generators for the rectangular $G_{2}$ are $\gamma_{1}=(I, \mathbf{a})$, $\gamma_{2}=(I, \mathbf{b})$, and $\gamma_{3}=(A, \mathbf{c})$, with $\mathbf{a}=L_{x} \hat{\mathbf{e}}_{x}, \mathbf{b}=L_{y} \hat{\mathbf{e}}_{y}$, $\mathbf{c}=2 \rho \cos \phi \hat{\mathbf{e}}_{x}+2 \rho \sin \phi \hat{\mathbf{e}}_{y}+L_{z} \hat{\mathbf{e}}_{z}$, and $A$ given in (D1) with $\alpha=\pi$. They satisfy the relations $\gamma_{1} \gamma_{3} \gamma_{1}=\gamma_{3}$ and $\gamma_{2} \gamma_{3} \gamma_{2}=\gamma_{3}$, which allow to write any isometry of the covering group by (D3) with

$$
\gamma_{3}^{n_{3}}= \begin{cases}\left(I, n_{3} \mathbf{c}_{\|}\right) & \text {if } n_{3} \text { is even } \\ \left(A, n_{3} \mathbf{c}_{\|}+\mathbf{c}_{\perp}\right) & \text { if } n_{3} \text { is odd, }\end{cases}
$$

where $\mathbf{c}_{\perp}=2 \rho \cos \phi \hat{\mathbf{e}}_{x}+2 \rho \sin \phi \hat{\mathbf{e}}_{y}$.

It follows from (D3) and (D4) that the expression (25) takes the form

$$
\begin{aligned}
Y_{\ell m}^{\Gamma}(\mathbf{k})= & \sum_{\mathbf{n} \in \mathbb{Z}^{3}} e^{-i\left(n_{x} k_{x} L_{x}+n_{y} k_{y} L_{y}+2 n_{z} k_{z} L_{z}\right)} \\
& \times\left[1+(-1)^{m} e^{-i \mathbf{k} \cdot \mathbf{c}}\right] Y_{\ell m}\left(\mathbf{n}_{\mathbf{k}}\right) \\
= & (2 \pi)^{3} \sum_{\mathbf{p} \in \mathbb{Z}^{3}} \delta\left(k_{x} L_{x}-2 \pi p_{x}\right) \delta\left(k_{y} L_{y}-2 \pi p_{y}\right) \\
& \times \delta\left(k_{z} L_{z}-\pi p_{z}\right) Y_{\ell m}\left(\mathbf{n}_{\mathbf{k}}\right) f_{m}^{\Gamma}(\mathbf{k}),
\end{aligned}
$$

where we have put $n_{1}=n_{x}, n_{2}=n_{y}$, and $n_{3}=2 n_{z}$ or $2 n_{z}+1$, depending on whether $n_{3}$ is even or odd. The components of $\vec{\beta}$ are $\beta_{x}=p_{x} / L_{x}, \beta_{y}=p_{y} / L_{y}$, and $\beta_{z}=$ $p_{z} / 2 L_{z}$.

\section{3. $G_{3}$}

The generators for a manifold of class $G_{3}$ are $\gamma_{1}=$ $(I, \mathbf{a}), \gamma_{2}=(I, \mathbf{b})$, and $\gamma_{3}=(A, \mathbf{c})$, with $\mathbf{a}=L \hat{\mathbf{e}}_{x}, \mathbf{b}=$ $-\frac{L}{2}\left(\hat{\mathbf{e}}_{x}-\sqrt{3} \hat{\mathbf{e}}_{y}\right), \quad \mathbf{c}=\frac{\rho}{2}(3 \cos \phi+\sqrt{3} \sin \phi) \hat{\mathbf{e}}_{x}+\frac{\rho}{2} \times$ $(3 \sin \phi-\sqrt{3} \cos \phi) \hat{\mathbf{e}}_{y}+L_{z} \hat{\mathbf{e}}_{z}$, and $A$ given in (D1) with $\alpha=2 \pi / 3$. They satisfy the relations $\gamma_{2}^{-1} \gamma_{3} \gamma_{1}=\gamma_{3}$ and $\gamma_{1} \gamma_{2} \gamma_{3} \gamma_{2}=\gamma_{3}$, which allow us to write any isometry of the covering group by (D3) with

$$
\gamma_{3}^{n_{3}}= \begin{cases}\left(I, n_{3} \mathbf{c}_{\|}\right) & \text {if } n_{3}=0 \bmod 3 \\ \left(A, n_{3} \mathbf{c}_{\|}+\mathbf{c}_{\perp}\right) & \text { if } n_{3}=1 \bmod 3 \\ \left(A^{2}, n_{3} \mathbf{c}_{\|}+\mathcal{O}_{2} \mathbf{c}_{\perp}\right) & \text { if } n_{3}=2 \bmod 3,\end{cases}
$$

where

$$
\mathbf{c}_{\perp}=\frac{\rho}{2}(3 \cos \phi+\sqrt{3} \sin \phi) \hat{\mathbf{e}}_{x}+\frac{\rho}{2}(3 \sin \phi-
$$

$\sqrt{3} \cos \phi) \hat{\mathbf{e}}_{y}$.

It follows from (D3) and (D5) that the expression (25) takes the form

$$
\begin{aligned}
\Upsilon_{\ell m}^{\Gamma}(\mathbf{k})= & \sum_{\mathbf{n} \in \mathbb{Z}^{3}} e^{-i\left\{n_{x} k_{x} L+n_{y}\left[(\sqrt{3 / 2}) k_{y}-(1 / 2) k_{x}\right] L+3 n_{z} k_{z} L_{z}\right\}} \\
& \times\left[1+\omega_{3}^{-m} e^{-i \mathbf{k} \cdot \mathbf{c}}+\omega_{3}^{-2 m} e^{-i \mathbf{k} \cdot \mathcal{O}_{2} \mathbf{c}}\right] Y_{\ell m}\left(\mathbf{n}_{\mathbf{k}}\right) \\
= & (2 \pi)^{3} \sum_{\mathbf{p} \in \mathbb{Z}^{3}} \delta\left(k_{x} L-2 \pi p_{x}\right) \delta\left(\left[\frac{\sqrt{3}}{2} k_{y}-\frac{1}{2} k_{x}\right] L\right. \\
& \left.-2 \pi p_{y}\right) \delta\left(k_{z} L_{z}-\frac{2 \pi}{3} p_{z}\right) Y_{\ell m}\left(\mathbf{n}_{\mathbf{k}}\right) f_{m}^{\Gamma}(\mathbf{k})
\end{aligned}
$$

where we have put $n_{1}=n_{x}, n_{2}=n_{y}$, and $n_{3}=3 n_{z}, 3 n_{z}+$ 1 or $3 n_{z}+2$ according to (D5). We also get $\beta_{x}=\frac{p_{x}}{L}, \beta_{y}=$ $[\sqrt{3} /(3 L)]\left(2 p_{y}+p_{x}\right)$, and $\beta_{z}=p_{z} /\left(3 L_{z}\right)$.

\section{4. $G_{4}$}

The generators for a manifold of class $G_{4}$ are $\gamma_{1}=$ $(I, \mathbf{a}), \gamma_{2}=(I, \mathbf{b})$, and $\gamma_{3}=(A, \mathbf{c})$, with $\mathbf{a}=L \hat{\mathbf{e}}_{x}, \mathbf{b}=$ $L \hat{\mathbf{e}}_{y}, \quad \mathbf{c}=\rho(\cos \phi+\sin \phi) \hat{\mathbf{e}}_{x}+\rho(\sin \phi-\cos \phi) \hat{\mathbf{e}}_{y}+$ $L_{z} \hat{\mathbf{e}}_{z}$, and $A$ given in (D1) with $\alpha=\pi / 2$. They satisfy the relations $\gamma_{2}^{-1} \gamma_{3} \gamma_{1}=\gamma_{3}$ and $\gamma_{1} \gamma_{3} \gamma_{2}=\gamma_{3}$, which allow us to write any isometry of the covering group by (D3) with

$$
\gamma_{3}^{n_{3}}= \begin{cases}\left(I, n_{3} \mathbf{c}_{\|}\right) & \text {if } n_{3}=0 \bmod 4 \\ \left(A, n_{3} \mathbf{c}_{\|}+\mathbf{c}_{\perp}\right) & \text { if } n_{3}=1 \bmod 4 \\ \left(A^{2}, n_{3} \mathbf{c}_{\|}+\mathcal{O}_{2} \mathbf{c}_{\perp}\right) & \text { if } n_{3}=2 \bmod 4 \\ \left(A^{3}, n_{3} \mathbf{c}_{\|}+\mathcal{O}_{3} \mathbf{c}_{\perp}\right) & \text { if } n_{3}=3 \bmod 4\end{cases}
$$

where $\mathbf{c}_{\perp}=\rho(\cos \phi+\sin \phi) \hat{\mathbf{e}}_{x}+\rho(\sin \phi-\cos \phi) \hat{\mathbf{e}}_{y}$.

Similarly, using (D3) and (D6), the expression (25) takes the form

$$
\begin{aligned}
Y_{\ell m}^{\Gamma}(\mathbf{k})= & \sum_{\mathbf{n} \in \mathbb{Z}^{3}} e^{-i\left(n_{x} k_{x} L+n_{y} k_{y} L+4 n_{z} k_{z} L_{z}\right)} \\
& \times\left[1+\sum_{h=1}^{3} \omega_{4}^{-h m} e^{-i \mathbf{k} \cdot \mathcal{O}_{h} \mathbf{c}}\right] Y_{\ell m}\left(\mathbf{n}_{\mathbf{k}}\right) \\
= & (2 \pi)^{3} \sum_{\mathbf{p} \in \mathbb{Z}^{3}} \delta\left(k_{x} L-2 \pi p_{x}\right) \delta\left(k_{y} L-2 \pi p_{y}\right) \\
& \times \delta\left(k_{z} L_{z}-\frac{\pi}{2} p_{z}\right) Y_{\ell m}\left(\mathbf{n}_{\mathbf{k}}\right) f_{m}^{\Gamma}(\mathbf{k})
\end{aligned}
$$

where we have put $n_{1}=n_{x}, n_{2}=n_{y}$, and $n_{3}=4 n_{z}, 4 n_{z}+$ $1,4 n_{z}+2$, or $4 n_{z}+3$ according to (D6). We also get $\beta_{x}=$ $\frac{p_{x}}{L}, \beta_{y}=\frac{p_{y}}{L}$, and $\beta_{z}=p_{z} /\left(4 L_{z}\right)$. 


\section{5. $\mathbf{G}_{5}$}

The generators for the rectangular $G_{5}$ are $\gamma_{1}=(I, \mathbf{a})$, $\gamma_{2}=(I, \mathbf{b})$, and $\gamma_{3}=(A, \mathbf{c})$, with $\mathbf{a}=L \hat{\mathbf{e}}_{x}, \mathbf{b}=\frac{L}{2}\left(\hat{\mathbf{e}}_{x}+\right.$ $\left.\sqrt{3} \hat{\mathbf{e}}_{y}\right), \quad \mathbf{c}=\frac{\rho}{2}(\cos \phi+\sqrt{3} \sin \phi) \hat{\mathbf{e}}_{x}+\frac{\rho}{2}(\sin \phi-$ $\sqrt{3} \cos \phi) \hat{\mathbf{e}}_{y}+L_{z} \hat{\mathbf{e}}_{z}$, and $A$ given in (D1) with $\alpha=\pi / 3$. They satisfy the relations $\gamma_{2}^{-1} \gamma_{3} \gamma_{1}=\gamma_{3}$ and $\gamma_{1} \gamma_{2}^{-1} \gamma_{3} \gamma_{2}=\gamma_{3}$, which allow us to write any isometry of the covering group by (D3) with

$$
\gamma_{3}^{n_{3}}= \begin{cases}\left(I, n_{3} \mathbf{c}_{\|}\right) & \text {if } n_{3}=0 \bmod 6 \\ \left(A, n_{3} \mathbf{c}_{\|}+\mathbf{c}_{\perp}\right) & \text { if } n_{3}=1 \bmod 6 \\ \left(A^{2}, n_{3} \mathbf{c}_{\|}+\mathcal{O}_{2} \mathbf{c}_{\perp}\right) & \text { if } n_{3}=2 \bmod 6 \\ \left(A^{3}, n_{3} \mathbf{c}_{\|}+\mathcal{O}_{3} \mathbf{c}_{\perp}\right) & \text { if } n_{3}=3 \bmod 6 \\ \left(A^{4}, n_{3} \mathbf{c}_{\|}+\mathcal{O}_{4} \mathbf{c}_{\perp}\right) & \text { if } n_{3}=4 \bmod 6 \\ \left(A^{5}, n_{3} \mathbf{c}_{\|}+\mathcal{O}_{5} \mathbf{c}_{\perp}\right) & \text { if } n_{3}=5 \bmod 6\end{cases}
$$

where $\mathbf{c}_{\perp}=\frac{\rho}{2}(\cos \phi+\sqrt{3} \sin \phi) \hat{\mathbf{e}}_{x}+\frac{\rho}{2}(\sin \phi-\sqrt{3} \cos \phi) \hat{\mathbf{e}}_{y}$.
Using (D3) and (D7), the expression (25) takes the form

$$
\begin{aligned}
Y_{\ell m}^{\Gamma}(\mathbf{k})= & \sum_{\mathbf{n} \in \mathbb{Z}^{3}} e^{-i\left\{n_{x} k_{x} L+n_{y}\left[(\sqrt{3} / 2) k_{y}+(1 / 2) k_{x}\right] L+6 n_{z} k_{z} L_{z}\right\}} \\
& \times\left[1+\sum_{h=1}^{5} \omega_{6}^{-h m} e^{-i \mathbf{k} \cdot \mathcal{O}_{h} \mathbf{c}}\right] Y_{\ell m}\left(\mathbf{n}_{\mathbf{k}}\right) \\
= & (2 \pi)^{3} \sum_{\mathbf{p} \in \mathbb{Z}^{3}} \delta\left(k_{x} L-2 \pi p_{x}\right) \delta\left(\left[\frac{\sqrt{3}}{2} k_{y}+\frac{1}{2} k_{x}\right] L\right. \\
& \left.-2 \pi p_{y}\right) \delta\left(k_{z} L_{z}-\frac{\pi}{3} p_{z}\right) Y_{\ell m}\left(\mathbf{n}_{\mathbf{k}}\right) f_{m}^{\Gamma}(\mathbf{k}),
\end{aligned}
$$

where we have put $n_{1}=n_{x}, n_{2}=n_{y}$, and $n_{3}=6 n_{z}, 6 n_{z}+$ $1,6 n_{z}+2, \ldots, 6 n_{z}+5$ according to (D7). We also get $\beta_{x}=\frac{p_{x}}{L}, \beta_{y}=[\sqrt{3} /(3 L)]\left(2 p_{y}-p_{x}\right)$, and $\beta_{z}=p_{z} /\left(6 L_{z}\right)$.
[1] M. Tegmark, A. de Oliveira-Costa, and A. J. S. Hamilton, Phys. Rev. D 68, 123523 (2003); A. de Oliveira-Costa, M. Tegmark, M. Zaldarriaga, and A. J. S. Hamilton, ibid. 69, 063516 (2004).

[2] C. J. Copi, D. Huterer, and G. D. Starkman, Phys. Rev. D 70, 043515 (2004); D. J. Schwarz, G. D. Starkman, D. Huterer, and C. J. Copi, Phys. Rev. Lett. 93, 221301 (2004); G. Katz and J.R. Weeks, Phys. Rev. D 70, 063527 (2004); K. Land and J. Magueijo, Mon. Not. R. Astron. Soc. 357, 994 (2005); 362, 838 (2005); Phys. Rev. Lett. 95, 071301 (2005).

[3] F. Lizhi and M. Houjun, Mod. Phys. Lett. A 2, 229 (1987); I. Yu. Sokolov, JETP Lett. 57, 617 (1993); A. A. Starobinsky, ibid. 57, 622 (1993); D. Stevens, D. Scott, and J. Silk, Phys. Rev. Lett. 71, 20 (1993); A. de OliveiraCosta and G. F. Smoot, Astrophys. J. 448, 477 (1995); A. de Oliveira-Costa, G. F. Smoot, and A. A. Starobinsky, ibid. 468, 457 (1996); astro-ph/9705125; N. J. Cornish, D. N. Spergel, and G. D. Starkman, Phys. Rev. D 57, 5982 (1998); N. J. Cornish and D. N. Spergel, ibid. 62, 087304 (2000); K. T. Inoue, in AIP Conference Proceedings " $3 K$ Cosmology”, edited by L. Maiani, F. Melchiorri, and N. Vittorio (American Institute of Physics, New York, 1999), p. 343; Prog. Theor. Phys. 106, 39 (2001); Classical Quantum Gravity 18, 1967 (2001); K. T. Inoue, K. Tomita, and N. Sugiyama, Mon. Not. R. Astron. Soc. 314, L21 (2000); J.-P. Luminet, J. Weeks, A. Riazuelo, R. Lehoucq, and J.-P. Uzan, Nature (London) 425, 593 (2003); J. Weeks, J.-P. Luminet, A. Riazuelo, and R. Lehoucq, Mon. Not. R. Astron. Soc. 352, 258 (2004); J. Gundermann, astro-ph/0503014.

[4] J. Levin, E. Scannapieco, and J. Silk, Phys. Rev. D 58, 103516 (1998); astro-ph/9811226.

[5] R. Aurich, Astrophys. J. 524, 497 (1999); R. Aurich and F. Steiner, Mon. Not. R. Astron. Soc. 323, 1016 (2001); R.
Aurich, S. Lustig, F. Steiner, and H. Then, Classical Quantum Gravity 21, 4901 (2004); R. Aurich, S. Lustig, and F. Steiner, ibid. 22, 2061 (2005); 22, 3443 (2005).

[6] K. T. Inoue, astro-ph/0011539; K. T. Inoue and N. Sugiyama, Phys. Rev. D 67, 043003 (2003).

[7] J. R. Weeks, astro-ph/0412231.

[8] N. J. Cornish, D. N. Spergel, and G. D. Starkman, Classical Quantum Gravity 15, 2657 (1998); J. R. Weeks, ibid. 15, 2599 (1998); B. F. Roukema, ibid. 17, 3951 (2000); Mon. Not. R. Astron. Soc. 312, 712 (2000); G. I. Gomero, astroph/0310749; B. F. Roukema, B. Lew, M. Cechowska, A. Marecki, and S. Bajtlik, Astron. Astrophys. 423, 821 (2004); J. Levin, Phys. Rev. D 70, 083001 (2004); 70, 083001 (2004); M. O. Calvão, G. I. Gomero, B. Mota, and M.J. Rebouças, Classical Quantum Gravity 22, 1991 (2005).

[9] N. J. Cornish, D. N. Spergel, G.D. Starkman, and E. Komatsu, Phys. Rev. Lett. 92, 201302 (2004).

[10] A. Hajian and T. Souradeep, astro-ph/0301590; Astrophys. J. 597, L5 (2003); Pramana 62, 793 (2004); astro-ph/ 0501001; A. Hajian, T. Souradeep, and N. Cornish, Astrophys. J. 618, L63 (2004).

[11] T. Souradeep and A. Hajian, astro-ph/0502248.

[12] J. Levin, J. D. Barrow, E. F. Bunn, and J. Silk, Phys. Rev. Lett. 79, 974 (1997); J. Levin, E. Scannapieco, G. de Gasperis, J. Silk, and J.D. Barrow, Phys. Rev. D 58, 123006 (1998).

[13] J. Levin, E. Scannapieco, and J. Silk, Classical Quantum Gravity 15, 2689 (1998).

[14] A. Riazuelo, J.-P. Uzan, R. Lehoucq, and J. Weeks, Phys. Rev. D 69, 103514 (2004).

[15] J.-P. Uzan, A. Riazuelo, R. Lehoucq, and J. Weeks, Phys. Rev. D 69, 043003 (2004).

[16] A. Riazuelo, J. Weeks, J.-P. Uzan, R. Lehoucq, and J.-P. Luminet, Phys. Rev. D 69, 103518 (2004). 
[17] J. R. Bond, D. Pogosyan, and T. Souradeep, in Proceedings of the 18th Texas Symposium on Relativistic Astrophysics, edited by A. Olinto, J. Frieman, and D. Schramm (World Scientific, Singapore, 1997); astro-ph/ 9804042; Classical Quantum Gravity 15, 2671 (1998); Phys. Rev. D 62, 043005 (2000); 62, 043006 (2000).

[18] R. Lehoucq, J. Weeks, J.-P. Uzan, E. Gausmann, and J.-P. Luminet, Classical Quantum Gravity 19, 4683 (2002); R. Lehoucq, J.-P. Uzan, and J. Weeks, Kodai Math. J. 26, 119 (2003); J. R. Weeks, math.SP/0502566.

[19] M. Lachièze-Rey, math.SP/0304409; Classical Quantum Gravity 21, 2455 (2004); math.SP/0401153; M. LachièzeRey and S. Caillerie, Classical Quantum Gravity 22, 695 (2005).

[20] N. J. Cornish and N. G. Turok, Classical Quantum Gravity 15, 2699 (1998); N. J. Cornish and D. N. Spergel, math.DG/9906017; K. T. Inoue, Classical Quantum Gravity 16, 3071 (1999); Phys. Rev. D 62, 103001 (2000); Classical Quantum Gravity 18, 629 (2001); R. Aurich, F. Steiner, and H. Then, gr-qc/0404020; R. Aurich, S. Lustig, F. Steiner, and H. Then, Phys. Rev. Lett. 94, 021301 (2005).

[21] G. I. Gomero, A.F.F. Teixeira, M. J. Reboucas, and A. Bernui, Int. J. Mod. Phys. D 11, 869 (2002); G. I. Gomero, M. J. Reboucas, and A. F. F. Teixeira, Phys. Lett. A 275, 355 (2000); Classical Quantum Gravity 18, 1885 (2001);
Int. J. Mod. Phys. D 9, 687 (2000).

[22] A.F. Beardon, The Geometry of Discrete Groups, Graduate Texts in Mathematics Vol. 91 (Springer, New York, 1983).

[23] G. I. Gomero, M. J. Rebouças, and R. Tavakol, Classical Quantum Gravity 18, 4461 (2001); 18, L145 (2001); Int. J. Mod. Phys. A 17, 4261 (2002); G. I. Gomero and M. J. Rebouças, Phys. Lett. A 311, 319 (2003); J. R. Weeks, R. Lehoucq, and J.-P. Uzan, Classical Quantum Gravity 20, 1529 (2003); J. R. Weeks, Mod. Phys. Lett. A 18, 2099 (2003); B. Mota, M. Makler, and M. J. Rebouças, astro-ph/ 0506499.

[24] B. Mota, G. I. Gomero, M. J. Rebouças, and R. Tavakol, Classical Quantum Gravity 21, 3361 (2004); M. Kunz, N. Aghanim, L. Cayon, O. Forni, A. Riazuelo, and J. P. Uzan, astro-ph/0510164.

[25] D. A. Varshalovich, A. N. Moskalev, and V. K. Khersonskii, Quantum Theory of Angular Momentum (World Scientific, Singapore, 1988).

[26] M. A. Blanco, M. Flórez, and M. Bermejo, J. Mol. Struct. 419, 19 (1997).

[27] J.A. Wolf, Spaces of Constant Curvature (Publish or Perish Inc., Delaware, 1984).

[28] G. I. Gomero and M. J. Rebouças, Phys. Lett. A 311, 319 (2003); G. I. Gomero, Classical Quantum Gravity 20, 4775 (2003). 